\title{
Simulation of Actively Controlled Spacecraft with Flexible Appendages
}

\author{
R. R. Ryan* \\ University of Michigan, Ann Arbor, Michigan
}

\begin{abstract}
Complex interplanetary spacecraft and newly proposed satellites for reconnaissance and strategic defense are being designed with increasingly light and flexible appendages despite ever more demanding requirements for accurate pointing and tracking. The onboard control systems required to satisfy these strict requirements during reasonably quiescent conditions must also act effectively to limit deformation and maintain stability of motion in the face of significant disturbances to flexible appendage motion resulting from spin-up, spin-down, orbital, and deployment maneuvers of various types. This paper incorporates a recently proposed theory for modeling flexible bodies into a framework for studying the effects of beamlike appendage deformation on overall system performance. llustrative examples involving use of the theory in practical applications are presented, and relative merits of this model theory vs nonlinear finite-element techniques are discussed.
\end{abstract}

\section{Introduction}

A NEW generation of spacecraft designs involving actively controlled systems with extremely light, flexible appendages has motivated increased research aimed at producing accurate models of such systems for purposes of simulation, structural verification, and control law design. A basic requirement of any such model intended for general simulation purposes is that it must be able to account properly for both large overall motions and concurrent small deformations of bodies, as well as to include accurately the important coupling effects existing between these two types of dynamic behavior.

Recently, a new technique ${ }^{1}$ was proposed for modeling the behavior of flexible beamlike appendages attached to a rigid base body undergoing large overall rotations and translations. The theory was developed by considering the base motion to be prescribed as a function of time; effects of the base motion on the small deformation of an elastic appendage were then studied using the new theory and more conventional ones. ${ }^{2-6}$ The study uncovered limitations of conventional flexible multibody formalisms applied to structural elements and provided the impetus for further development and implementation of the theory into a framework for analyzing free-flying bodies wherein effects of flexible appendage motion on uncontrolled and actively controlled overall system motion could be investigated.

The present paper has a threefold purpose. First, in order to extend the theory to deal with free-flying systems without prescribed base motion, the requisite additional kinematical and dynamical equations governing motion of the base are presented, and techniques for incorporating general external forcing effects; such as gravitational attraction and control system actuation, are discussed. Second, in order to illustrate the effectiveness of the model in analyzing spacecraft, simulation results for a few representative problems will be shown, and the effect of the flexible appendage motion on overall base motion will be highlighted in contrast to the aforemen-

Presented as Paper AAS 87-0478 at the AAS/AIAA Astrodynamics Specialist Conference, Kalispell, MT, Aug. 11, 1987; received Nov. 2, 1987; revision received March 14, 1988. Copyright (C) 1988 by the American Institute of Aeronautics and Astronautics, Inc. All rights reserved.

*Professor, Department of Mechanical Engineering and Applied Mechanics; currently, Vice President, Mechanical Dynamics, Ann Arbor, MI. tioned work, which dealt primarily with the effect of base motion on appendage behavior. Last, the enhanced modal approach, on which this work is based, will be compared with nonlinear finite-element formulations aimed at solution of similar problems; relative efficiency, accuracy, and ease of implementation will be discussed.

The remainder of this paper is organized as follows. The system to be studied is described in detail in the next section. In Sec. III, the theory presented in Ref. 1 is extended to the free-flying case, and supplementary equations of motion are given. Illustrative examples showing general use of the theory and equations in practical applications are included in Sec. IV. The last section concludes with a discourse on the relative advantages and disadvantages of employing enhanced modal techniques rather than nonlinear finite-element procedures to perform simulations of complex deformable spacecraft undergoing general maneuvers.

\section{System Description}

The system to be analyzed, shown in Fig. 1, consists of a flexible beam $B$, fixed at one end to a rigid body $A$, which is capable of undergoing large three-dimensional translations and rotations in a Newtonian reference frame $N$. The rigid base is characterized by its mass $m_{A}$ and six independent inertia parameters $J_{11}, J_{12}, J_{13}, J_{22}, J_{23}$, and $J_{33}$, which are

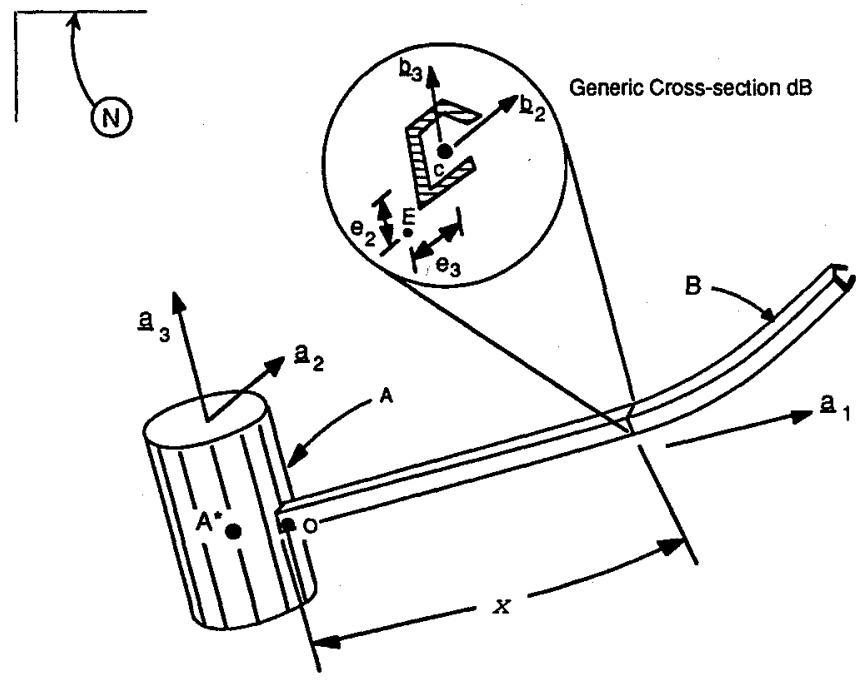

Fig. 1 Satellite with deformable beamlike appendage. 
measure numbers of the central inertia dyadic $\mathbf{J}$ of $A$ expressed in terms of a dextral set of mutually perpendicular unit vectors $a_{1}, a_{2}, a_{3}$, fixed in $A$, and directed as shown. That is,

$$
\begin{aligned}
\underline{\mathbf{J}} & =J_{11} a_{1} a_{1}+J_{12} a_{1} a_{2}+J_{13} a_{1} a_{3}+J_{12} a_{2} a_{1}+J_{22} a_{2} a_{2} \\
& +J_{23} a_{2} a_{3}+J_{13} a_{3} a_{1}+J_{23} a_{3} a_{2}+J_{33} a_{3} a_{3}
\end{aligned}
$$

The beam is characterized by a natural length $L$, material properties $E_{o}(x), G(x), \rho(x)$, and cross-sectional properties $A_{o}(x), I_{2}(x), I_{3}(x), \alpha_{2}(x), \alpha_{3}(x), \kappa(x), \Gamma(x), e_{2}(x)$, and $e_{3}(x)$, defined as follows. Let $x$ be the distance from a point $O$, located at the root of $B$ to the plane of an arbitrary cross section of $B$, when $B$ is undeformed. Then $E_{o}(x), G(x)$, and $\rho(x)$ are the beam's modulus of elasticity, shear modulus, and mass per unit length, respectively, each a function of $x$. The area of the cross section located at a distance $x$ from point $O$ is denoted as $A_{o}(x)$, and the Saint Venant torsion factor and the warping factor are represented by the symbols $\kappa(x)$ and $\Gamma(x)$, respectively. In order to define the symbols $I_{2}(x), I_{3}(x)$, $\alpha_{2}(x), \alpha_{3}(x), e_{2}(x)$, and $e_{3}(x)$, introduce a dextral set of mutually perpendicular unit vectors $b_{1}, b_{2}, b_{3}$, fixed in the plane of the cross section located at a distance $x$ from point $O$ and oriented such that $b_{1}$ is parallel to the centroidal axis of $B$, while $b_{2}$ and $b_{3}$ are parallel to central principal axes of the cross section. Unit vectors $a_{1}, a_{2}, a_{3}$ are parallel, respectively, to $b_{1}, b_{2}, b_{3}$ prior to deformation of $B$. The symbols $I_{2}(x)$ and $I_{3}(x)$ denote the central principal second moments of area of the generic cross section for unit vectors $b_{2}$ and $b_{3}$, respectively, and $\alpha_{2}(x)$ and $\alpha_{3}(x)$ are the shear area ratios (also called shear correction factors; see Ref. 7, p. 351) of the section for the $b_{2}$ and $b_{3}$ directions. The parameters $e_{2}(x)$ and $e_{3}(x)$ are measure numbers of the $b_{2}$ and $b_{3}$ components of the eccentricity vector, which extends from the elastic center of the generic cross section to the centroid. The elastic center, the flexural center, and the center of twist are all assumed to coincide in the present analysis. ${ }^{8}$ Lastly, the location of the mass center $A^{*}$ of $A$, relative to point $O$, is specified in terms of three scalar quantites $d_{1}, d_{2}$, and $d_{3}$ representing the $a_{1}, a_{2}$, $a_{3}$ measure numbers, respectively, of the position vector extending from $O$ to $A^{*}$.

\section{Equations of Motion}

Reference 1 also treats the system described in Sec. II and contains a detailed discussion of equations of motion applicable when the base motion is prescribed as a function of time. In that regard, a geometric constraint, similar to that proposed by Hughes and Fung, ${ }^{16}$ was included in the formulation to account properly for the interrelationship of axial and bending deformation in long, thin structural elements, and an algorithm was presented for analyzing general beams attached to moving bases. The treatment of beam deformation using the embedded constraint approach quite naturally leads to dynamical equations that are totally linear in the deformation variables and yet properly account for motion-induced stiffness variations as pointed out by Kaza and Kvaternik. ${ }^{17}$ The present section is intended to supplement the previous equations with additional equations needed to treat three-dimensional free base motion and arbitrary external forcing functions such as might arise from gravitational attraction and active control systems. As before, the interdependencies of orthogonal beam displacements will be properly taken into account in the development of equation terms that represent the coupling effects between flexible appendage motion and large rigidbody base motion.

To keep the number of equations to a minimum, terms and expressions developed explicitly in Ref. 1 will, in general, not be redeveloped or rewritten here. Enough detail will be given, however, so that the two papers together provide a complete set of equations and parameter definitions to allow for total understanding as well as construction of algorithms for the simulation of motions of systems consisting of a rigid base portion and one or more flexible beamlike appendages.

In the previous analysis, the motion of the base $A$ was characterized in terms of six scalar quantities $\omega_{1}, \omega_{2}, \omega_{3}, v_{1}$, $v_{2}$, and $v_{3}$, with $\omega_{1}, \omega_{2}, \omega_{3}$ defined as the $a_{1}, a_{2}, a_{3}$ measure numbers of the inertial angular velocity vector, ${ }^{N} \omega^{A}$, of rigid body $A$, and $v_{1}, v_{2}$, and $v_{3}$ defined as the $a_{1}, a_{2}, a_{3}$ measure numbers of the translational velocity ${ }^{N} v o$, of point $O$, on the elastic axis of $B$ at the root, such that

$$
{ }^{N} \omega^{A}=\omega_{1} a_{1}+\omega_{2} a_{2}+\omega_{3} a_{3}
$$

and

$$
{ }^{v^{0}}=v_{1} a_{1}+v_{2} a_{2}+v_{3} a_{3}
$$

(The elastic axis is the line along which transverse loads must be applied in order to produce bending unaccompanied by torsion of the beam at any section. ${ }^{8}$ This axis passes through the elastic center $E$ of every section.)

The deformation of $B$, on the other hand, was described in terms of assumed modal functions $\phi_{j i}(x)(j=1, \ldots, 6 ; i=$ $1, \ldots, v)$ and generalized coordinates $q_{i}(i=1, \ldots, v)$ in such a way that the elastic axis stretch $s(x, t)$, the two transverse shear center displacements $u_{2}(x, t)$ and $u_{3}(x, t)$, and the three successive rotation angles (see Ref. 1) $\theta_{i}(x, t)(i=1,2,3)$ of a particular cross section, located at a distance $x$ from $O$, could be written

$$
\begin{array}{ll}
s(x, t)=\sum_{i=1}^{v} \phi_{1 i}(x) q_{i}(t) & \\
u_{j}(x, t)=\sum_{i=1}^{v} \phi_{j i}(x) q_{i}(t) & (j=2,3) \\
\theta_{j}(x, t)=\sum_{i=1}^{v} \phi_{j+3, i}(x) q_{i}(t) & (j=1,2,3)
\end{array}
$$

The symbol $v$ in these equations represents the integer number of terms retained in the modal series. With $v$ deformational degrees of freedom and six rigid-body degrees of freedom of $A$, it is necessary to form at least $v+6$ dynamical first-order differential equations and $v+6$ kinematical first-order differential equations in order to determine completely the configuration and state of the system at any instant in time. To facilitate the formation of dynamical equations in the next section, it is beneficial to introduce new quantities, $u_{i}^{*}$ $(i=1, \ldots, v+6)$, referred to as generalized speeds (Ref. 9, p. 87). These are defined as

$$
u_{i}^{*}\left\{\begin{array}{cl}
\dot{q}_{i} & (i=1, \ldots, v) \\
\triangleq \omega_{i-v} & (i=v+1, \ldots, v+3) \\
v_{i-v-3} & (i=v+4, \ldots, v+6)
\end{array}\right.
$$

\section{Dynamical Equations}

The dynamical equations can be written in the form

$$
F_{i}^{*}+F_{i}=0 \quad(i=1, \ldots, v+6)
$$

where $F_{i}^{*}$ and $F_{i}$ are the generalized inertia force and generalized active force, respectively, associated with the $i$ th generalized speed $u_{i}^{*}$ of the system. Before these generalized forces are given explicitly, it is necessary to form additional kinematic expressions.

The inertial velocity of $A^{*}$, the mass center of $A$, can be described in terms of the velocity of point $O$ and the angular velocity of $A$ by using the relationship

$$
{ }^{N} \boldsymbol{v}^{A^{*}}={ }^{N} v^{O}+{ }^{N} \omega^{A} \times p^{O A^{*}}
$$


where $p^{O A^{*}}$ is the position vector from $O$ to $A^{*}$, expressed in terms of three (not necessarily positive) scalar quantities $d_{1}$, $d_{2}$, and $d_{3}$, such that

$$
p^{o A^{*}} \triangleq d_{1} a_{1}+d_{2} a_{2}+d_{3} a_{3}
$$

Hence, it follows from Eqs. (1), (2), (8), and (9) that

$$
\begin{aligned}
& { }^{\boldsymbol{v}_{\boldsymbol{v}} A^{*}}=\left(v_{1}+\omega_{2} d_{3}-\omega_{3} d_{2}\right) \boldsymbol{a}_{1}+\left(v_{2}+\omega_{3} d_{1}-\omega_{1} d_{3}\right) \boldsymbol{a}_{2} \\
& \quad+\left(v_{3}+\omega_{1} d_{2}-\omega_{2} d_{1}\right) a_{3}
\end{aligned}
$$

Terms referred to as partial velocities of $A^{*}$ in $N$ and partial angular velocities of $A$ in $N$ are denoted by the symbols ${ }^{N} v_{i}^{A^{*}}$ and ${ }^{N} \omega_{i}^{A}(i=1, \ldots, v+6)$, respectively. Together with the partial velocities of $C$ in $N,{ }^{N} \boldsymbol{v}_{i}^{C}$, and partial angular velocities of $\mathrm{d} B$ in $N,{ }^{N} \omega_{i}^{\mathrm{d} B}$, introduced in the earlier analysis, they play a major role in the formulation of equations of motion. Note that $\mathrm{d} B$ is a differential element of $B$, and $C$ is the centroid of this element. The partial velocity ${ }^{N^{*} \boldsymbol{A}_{i}^{*}}$ is defined as the coefficient of the $i$ th generalized speed $u_{i}^{*}$ $(i=1, \ldots, v+6)$ in the velocity expression in Eq. (10), and ${ }^{N} \omega_{i}^{A}$ is defined as the coefficient of the $i$ th generalized speed in the angular velocity expression given in Eq. (1). Hence,

$$
\begin{aligned}
& { }^{N} \boldsymbol{v}_{i}^{A^{*}}={ }^{N} \boldsymbol{\omega}_{i}^{A}=0 \quad(i=1, \ldots, v) \\
& N_{v_{v+i}^{A^{*}}}^{A^{*}}= \begin{cases}\epsilon_{i j k} d \boldsymbol{a}_{k} & (i=1,2,3) \\
\boldsymbol{a}_{i-3} & (i=4,5,6)\end{cases} \\
& { }^{N} \omega_{v+i}^{A}= \begin{cases}a_{i} & (i=1,2,3) \\
0 & (i=4,5,6)\end{cases}
\end{aligned}
$$

where $\epsilon_{i j k}$ is the Levi-Civita permutation symbol, $\dagger$ and a summation convention is employed. (According to this convention, a repeated subscript of $i, j, k, l$, or $m$ indicates that summation as the index that is repeated takes the values of $1,2,3$, while a repeated subscript $n$ indicates summation as $n$ takes the values of $1, \ldots, \nu$.) The linearized partial velocities $N_{\tilde{\boldsymbol{v}}}^{C}$ and partial angular velocities ${ }^{N} \tilde{\boldsymbol{\omega}}_{i}^{\mathrm{d} B}$ have been given explicitly in Ref. 1 for $i=1, \ldots, \nu$. After appropriate linearization in

$$
N_{\tilde{v}_{a \nu+j}}^{C}=\left\{\begin{array}{cc}
\boldsymbol{a}_{i} \epsilon_{i j k}\left[\delta_{k l} \phi_{l n} q_{n}+x \delta_{k 1}+\delta_{k l}\left(1-\delta_{1 l}\right) e_{l}\right. & \\
\left.+\epsilon_{k l m} \phi_{l+3, n} q_{n} e_{m}\left(1-\delta_{m 1}\right)\right] & (j=1,2,3) \\
a_{j-3} & (j=4,5,6)
\end{array}\right.
$$

and

$$
{ }^{N} \tilde{\omega}_{v+j}^{d B}= \begin{cases}b_{i}\left[\delta_{i j}+\epsilon_{j k i} \phi_{k+3, n} q_{n}\right] & (j=1,2,3) \\ 0 & (j=4,5,6)\end{cases}
$$

The symbol $\delta_{i j}$ denotes the Kronecker delta, which is equal to unity when the subscripts $i$ and $j$ have the same value and otherwise is equal to zero.

With the partial velocities and partial angular velocities defined as in the preceding paragraph, the generalized inertia forces for the system composed of bodies $A$ and $B$ appear as

$$
\begin{aligned}
F_{i}^{*} & ={ }^{N} \boldsymbol{v}_{i}^{A^{*}} \cdot\left(-m_{A}{ }^{N} a^{A^{*}}\right)-{ }^{N} \omega_{i}^{A} \cdot\left({ }^{N} \boldsymbol{\alpha}^{A} \cdot \underline{\mathbf{J}}+{ }^{N} \omega^{A} \times \underline{\mathbf{J}} \cdot{ }^{N} \omega^{A}\right) \\
& -\int_{0}^{L}{ }^{N} \tilde{v}_{i}^{C} \cdot{ }^{N} \tilde{\boldsymbol{a}}^{C} \rho \mathrm{d} x-\int_{0}^{L}{ }^{N} \tilde{\omega}_{i}^{\mathrm{d} B} \\
& \cdot\left({ }^{N} \tilde{\boldsymbol{\alpha}}^{\mathrm{d} B} \cdot \underline{\mathrm{I}}+{ }^{N} \tilde{\boldsymbol{\omega}}^{\mathrm{d} B} \times \underline{\mathbf{I}} \cdot{ }^{N} \tilde{\boldsymbol{\omega}}^{\mathrm{d} B}\right) \rho \mathrm{d} x \quad(i=1, \ldots, v+6)
\end{aligned}
$$

†The quantity $\epsilon_{i j k}$ can be expressed as

$$
\epsilon_{i j k}=\frac{1}{2}(i-j)(j-k)(k-i) \quad(i, j, k=1,2,3)
$$

where ${ }^{N} a^{A^{*}}$ is the inertial acceleration of the mass center of $A$, ${ }^{N} \alpha^{A}$ the inertial angular acceleration of $A$ in $N,{ }^{N} \tilde{\omega}^{\mathrm{d} B}$ the linearized inertial angular velocity of a generic differential element $\mathrm{d} B$ of $B,{ }^{N} \tilde{\boldsymbol{a}}^{C}$ the linearized inertial acceleration of the centroid of a generic differential element located at a distance $x$ from $O,{ }^{N} \tilde{\boldsymbol{\alpha}}^{\mathrm{d} B}$ the linearized angular acceleration in $N$ of a differential element of $B, \mathrm{I} \mathrm{d} x$ the central inertia dyadic of $\mathrm{d} B$, and all other terms have been previously defined. The dyadic $\underline{I}$ can be represented as $\underline{I}=\rho\left(I_{11} b_{1} b_{1}+I_{22} b_{2} b_{2}+I_{33} b_{3} b_{3}\right)$ where $I_{11}, I_{22}, I_{33}$, expressed in terms of the central principal second moments of area $I_{2}$ and $I_{3}$, are given by

$$
I_{22} \triangleq \frac{I_{2}}{A_{o}}, \quad I_{33} \triangleq \frac{I_{3}}{A_{o}}, \quad I_{11} \triangleq \frac{I_{2}+I_{3}}{A_{o}}=I_{22}+I_{33}
$$

It is important to note that whereas quantities related to the deformation of $B$ have been linearized in the generalized coordinates $q_{1}, \ldots, q_{v}$ and in the generalized speeds $u_{1}^{*}, \ldots, u_{v}^{*}$, no quantities have been linearized in the variables $u_{v+1}^{*}, \ldots, u_{v+6}^{*}$, which characterize the arbitrarily large base motion. The acceleration ${ }^{N} a^{A^{*}}$ and angular acceleration ${ }^{N} \alpha^{A}$ can be derived in a straightforward manner by temporal differentiation, in $N$, of the velocity and angular velocity expressions appearing in Eqs. (10) and (1), respectively.

The system generalized active forces appear in their most complete form as

$$
\begin{aligned}
& F_{i}={ }^{N}{ }_{\boldsymbol{v}}^{A_{i}^{*}} \cdot(\boldsymbol{R})^{A^{*}}+{ }^{N} \boldsymbol{\omega}_{i}^{A} \cdot(\boldsymbol{T})^{A} \\
&+{ }^{N} \tilde{\boldsymbol{v}}_{i}^{C}(x=\sigma) \cdot(\boldsymbol{R})_{x=\sigma}^{C}+{ }^{N} \tilde{\boldsymbol{\omega}}_{i}^{\mathrm{d} B}(x=\sigma) \cdot(\boldsymbol{T})_{x=\sigma}^{\mathrm{d} B} \\
&+\int_{0}^{L}{ }^{N} \tilde{\boldsymbol{v}}_{i}^{C} \cdot(\boldsymbol{R})^{C} \mathrm{~d} x+\int_{0}^{L}{ }^{N} \tilde{\boldsymbol{\omega}}_{i}^{\mathrm{d} B} \cdot(\boldsymbol{T})^{\mathrm{d} B} \mathrm{~d} x \\
&(i=1, \ldots, v+6)
\end{aligned}
$$

where the quantities $(R)^{A^{*}},(R)_{x=\sigma}^{C},(R)^{C}$, and $(T)^{A},(T)_{x=\sigma}^{\mathrm{d} B}$, $(T)^{\mathrm{d} B}$ are defined as follows. Consider the system of all forces and torques acting on $A$ to be replaced with a couple of torque $(T)^{A}$ and a resultant force $(\boldsymbol{R})^{A^{*}}$ whose line of action passes through $A^{*}$. Furthermore, assume that all of the forces acting on a particular cross section $\mathrm{d} B$ of $B$ can be replaced with a couple of torque $(T)^{\mathrm{d} B}$ applied to $\mathrm{d} B$ and a resultant $(\boldsymbol{R})^{C}$ whose line of action passes through the centroid $C$ of the section. When forces are distributed along the length of the beam, then $(T)^{\mathrm{d} B}$ and $(\boldsymbol{R})^{C}$ are to be treated as functions of $x$, as shown in the third line of Eq. (18). Alternatively, when a set of forces is concentrated over a particular cross section at a distance $x=\sigma$ from point $O$, then $(T)^{\mathrm{d} B}$ and $(R)^{C}$ at that cross section are denoted as $(\boldsymbol{T})_{x=\sigma}^{\mathrm{d} B}$ and $(\boldsymbol{R})_{x=\sigma}^{C}$, and their contribution to the generalized active forces is determined as shown in line 2 of Eq. (18). All of these active forces and torques can be viewed as consisting of two distinct contributions; that is, a contribution due to internal forces arising from elastic strains and a contribution from external forces, including contact forces (e.g., friction forces, actuator forces) and distance or body forces (e.g., gravitational forces, drag forces, magnetic forces). The external force contribution to the generalized active forces will be treated subsequently; at present, only internal forces will be considered. The contribution of these internal forces was given in Ref. 1 for the case in which the motion of $A$ is prescribed as a function of time. Allowing $A$ to move freely does not alter this result. Hence,

$\left(F_{i}\right)_{\text {internal }}= \begin{cases}-\sum_{j=1}^{v} H_{i j} q_{j} & (i=1, \ldots, v) \\ 0 & (i=v+1, \ldots, v+6)\end{cases}$

where $H_{i j}$ is equivalent to $\partial^{2} U / \partial q_{i} \partial q_{j}$, with $U$ representing the system strain energy. 
Once the operations indicated in Eq. (16) have been carried out and the result has been substituted along with the quantity $F_{i}(i=1, \ldots, v+6)$ from Eq. (18), into Eq. (7), one arrives at the $v+6$ first-order dynamical equations, which can be expressed in matrix form as

$$
\mathscr{M} \dot{\mathbf{u}}^{*}+\mathscr{G} \mathbf{u}^{*}+\mathscr{K} \mathbf{q}=\mathscr{F}
$$

where $\mathscr{M}, \mathscr{G}$, and $\mathscr{K}$ are mass, gyroscopic, and stiffness matrices, respectively, all of size $(v+6) \times(v+6), \mathscr{F}$ is a $(v+6) \times 1$ column matrix of force terms, $\dot{\mathbf{u}}^{*}$ and $\mathbf{u}^{*}$ are $(v+6) \times 1$ column matrices with $\dot{u}_{i}^{*}$ and $u_{i}^{*}$ as the respective $i$ th elements, and $q$ is a $(v+6) \times 1$ column matrix with element $q_{i}$ in the $i$ th row for $i=1, \ldots, v$ and zero in the $i$ th row for $i=v+1, \ldots, v+6$. The matrices $\mathscr{M}, \mathscr{G}, \mathscr{K}$, and $\mathscr{F}$ appear in partitioned form as

$$
\begin{array}{cc}
\mathfrak{N}=\left[\begin{array}{c:c:c}
\mathbf{M} & \overline{\mathbf{M}}^{T}+\mathbf{M}^{*} \\
\hdashline \mathbf{M} & \mathbf{\mathbf { M }}
\end{array}\right] \quad \mathcal{S}=\left[\begin{array}{c:c}
\mathbf{G} & 0 \\
\hdashline 0 & 0
\end{array}\right] \\
\mathscr{K}=\left[\begin{array}{c:c}
\mathbf{K}-\mathbf{K}^{*} & 0 \\
\hdashline 0 & 0
\end{array}\right] \quad \mathscr{F}=\left[\begin{array}{c}
\mathbf{F}+\overline{\mathbf{F}} \\
\hdashline \overline{\mathbf{F}}
\end{array}\right]
\end{array}
$$

where $\mathbf{M}_{2} \mathbf{G}, \mathbf{K}$, and $\mathbf{F}$ are the matrices given in Ref. 1, whereas $\overline{\mathbf{M}}, \mathbf{M}^{*}, \overline{\mathbf{M}}, \mathbf{K}^{*}, \overline{\mathbf{F}}$, and $\overline{\mathbf{F}}$ are new matrices of size $6 \times v, v \times 6,6 \times 6, v \times v, v \times 1$, and $6 \times 1$, respectively, and will be given in explicit form presently. (To maximize clarity and conciseness, all terms involving beam eccentricities have been dropped from the analysis. To include them, one simply needs to augment the kinematic quantities in the equation of motion with the $e_{2}$ and $e_{3}$ terms as shown in Ref. 1.) First, however, it is convenient to introduce five symbols, $m_{B}, P_{1}$, $P_{2}, I_{B 2}$, and $I_{B 3}$, to represent new modal integrals, in addition to the modal integrals $W_{k l i j}, \breve{X}_{k i}, X_{k l i j}, \breve{Y}_{k i}, Y_{k l i j}, \breve{Z}_{k i}, Z_{k l i j}, \mu_{i j}$, and $\eta_{i j}(i, j=1, \ldots, v ; k, l=1, \ldots, 6)$ introduced in the previous analysis.

$$
\begin{gathered}
m_{B}=\int_{0}^{L} \rho \mathrm{d} x \\
P_{1}=\int_{0}^{L} \rho x \mathrm{~d} x, \quad P_{2}=\int_{0}^{L} \rho x^{2} \mathrm{~d} x \\
I_{B 2}=\int_{0}^{L} \rho I_{22} \mathrm{~d} x, \quad I_{B 3}=\int_{0}^{L} \rho I_{33} \mathrm{~d} x
\end{gathered}
$$

The elements of $\breve{\mathbf{M}}$ and $\mathbf{M}^{*}$, namely, $\breve{M}_{i j}$ and $M_{j i}^{*}(i=1, \ldots, 6$; $j=1, \ldots, v)$, are given as

$$
\begin{aligned}
& \check{M}_{1 j}=\breve{Y}_{4 j}+\breve{Z}_{4 j}, \quad \check{M}_{2 j}=-\breve{X}_{3 j}+\check{Y}_{5 j} \quad(j=1, \ldots, v) \\
& \breve{M}_{3 j}=\breve{X}_{2 j}+\breve{Z}_{6 j}, \quad \breve{M}_{4 j}=\breve{W}_{1 j} \quad(j=1, \ldots, v) \\
& \breve{M}_{5 j}=\breve{W}_{2 j}, \quad \breve{M}_{6 j}=\breve{W}_{3 j} \quad(j=1, \ldots, v)
\end{aligned}
$$

and

$$
\begin{aligned}
& M_{i 1}^{*}=\sum_{j=1}^{v}\left\{W_{32 i j}-W_{23 i j}\right. \\
& \left.-\left(Y_{56 i j}+Y_{65 i j}\right)\right\} q_{j} \quad(i=1, \ldots, v) \\
& M_{i 2}^{*}=\sum_{j=1}^{v}\left\{W_{13 i j}-W_{31 i j}+\left(Y_{46 i j}+Y_{64 i j}\right)\right. \\
& \left.+\left(Z_{46 i j}-Z_{64 i j}\right)\right\} q_{j} \quad(i=1, \ldots, v) \\
& M_{i 3}^{*}=\sum_{j=1}^{\nu}\left\{W_{21 i j}-W_{12 i j}-\left(Y_{45 i j}-Y_{54 i j}\right)\right. \\
& \left.-\left(Z_{45 i j}+Z_{54 i j}\right)\right\} q_{j} \\
& (i=1, \ldots, v) \\
& M_{i 4}^{*}=\sum_{j=1}^{v}\left[-\mu_{i j}\right] q_{j}, \quad M_{i 5}^{*}=M_{i 5}^{*}=0 \quad(i=1, \ldots, v)
\end{aligned}
$$

The matrix $\overline{\overline{\mathbf{M}}}$ is symmetric (i.e., $\overline{\bar{M}}_{i j}=\overline{\bar{M}}_{j i}$, for $i, j$ $=1, \ldots, 6) ;$ therefore, only the terms $\bar{M}_{i j} \quad(i=1, \ldots, 6$; $j=i, \ldots, 6)$ will be recorded. Specifically,

$$
\begin{aligned}
& \bar{M}_{11}=m_{A}\left(d_{2}^{2}+d_{3}^{2}\right)+J_{11}+\left(I_{B 2}+I_{B 3}\right) \\
& \bar{M}_{12}=-m_{A} d_{1} d_{2}+J_{21}+\sum_{j=1}^{v}\left[-\check{X}_{2 j}+\check{Z}_{6 j}\right] q_{j} \\
& \bar{M}_{13}=-m_{A} d_{1} d_{3}+J_{31}-\sum_{j=1}^{\nu}\left[\breve{X}_{3 j}+\breve{Y}_{5 j}\right] q_{j} \\
& \bar{M}_{14}=\bar{M}_{25}=\bar{M}_{36}=\bar{M}_{45}=\bar{M}_{46}=\bar{M}_{56}=0 \\
& \bar{M}_{15}=-m_{A} d_{3}-\sum_{j=1}^{v}\left[\breve{W}_{3 j}\right] q_{j} \\
& \bar{M}_{16}=m_{A} d_{2}+\sum_{j=1}^{v}\left[\breve{W}_{2 j}\right] q_{j} \\
& \bar{M}_{22}=m_{A}\left(d_{1}^{2}+d_{3}^{2}\right)+J_{22}+P_{2}+I_{B 2}+2 \sum_{j=1}^{v}\left[\breve{X}_{1 j}\right] q_{j} \\
& \bar{M}_{23}=-m_{A} d_{2} d_{3}+J_{32}+\sum_{j=1}^{v}\left[\check{Y}_{4 j}-\check{Z}_{4 j}\right] q_{j} \\
& \bar{M}_{24}=m_{A} d_{3}+\sum_{j=1}^{v}\left[\breve{W}_{3 j}\right] q_{j} \\
& \bar{M}_{26}=-m_{A} d_{1}-P_{1}-\sum_{j=1}^{v}\left[\breve{W}_{1 j}\right] q_{j} \\
& \bar{M}_{33}=m_{A}\left(d_{1}^{2}+d_{2}^{2}\right)+J_{33}+P_{2}+I_{B 3}+2 \sum_{j=1}^{v}\left[\breve{X}_{1 j}\right] q_{j} \\
& \bar{M}_{34}=-m_{A} d_{2}-\sum_{j=1}^{v}\left[\breve{W}_{2 j}\right] q_{j} \\
& \bar{M}_{35}=m_{A} d_{1}+P_{1}+\sum_{j=1}^{v}\left[\breve{W}_{1 j}\right] q_{j} \\
& \bar{M}_{44}=\bar{M}_{55}=\bar{M}_{66}=m_{A}+m_{B}
\end{aligned}
$$

The matrix $\mathbf{K}^{*}$, with elements $K_{i j}^{*}$, is related to the matrix $\mathrm{M}^{*}$, with elements $M_{i j}^{*}$, in the following way:

$$
\sum_{j=1}^{v} K_{i j}^{*} q_{j}=\sum_{j=1}^{6} M_{i j}^{*} \dot{u}_{v+j}^{*} \quad(i=1, \ldots, v)
$$

Hence, explicit $K_{i j}^{*}$ terms can be found directly from the terms in Eqs. (29-32).

The element $\breve{F}_{i}$ in the $i$ th row of the matrix is defined as

$$
\breve{F}_{i} \triangleq \sum_{j=1}^{6} \breve{M}_{j i} \dot{u}_{j+v}^{*} \quad(i=1, \ldots, v)
$$

while the elements of $\overline{\mathbf{F}}$, namely, $\bar{F}_{i}(i=1, \ldots, 6)$, appear as

$$
\begin{aligned}
\bar{F}_{1} & =m_{A}\left\{d_{3}\left[\omega_{3}\left(v_{1}+\omega_{2} d_{3}-\omega_{3} d_{2}\right)-\omega_{1}\left(v_{3}+\omega_{1} d_{2}-\omega_{2} d_{1}\right)\right]\right. \\
& \left.+d_{2}\left[\omega_{2}\left(v_{1}+\omega_{2} d_{3}-\omega_{3} d_{2}\right)-\omega_{1}\left(v_{2}+\omega_{3} d_{1}-\omega_{1} d_{3}\right)\right]\right\} \\
& +\omega_{2} \omega_{3}\left(J_{22}-J_{33}\right)+\omega_{3} \omega_{1} J_{21}+\omega_{3}^{2} J_{23} \\
& -\omega_{1} \omega_{2} J_{31}-\omega_{2}^{2} J_{32}+\left(I_{B 2}-I_{B 3}\right) \omega_{2} \omega_{3} \\
& +\sum_{j=1}^{v}\left\{\left[\breve{W}_{3 j}\left(\omega_{3} v_{1}-\omega_{1} v_{3}\right)+\breve{X}_{3 j} \omega_{1} \omega_{2}-\breve{X}_{2 j} \omega_{1} \omega_{3}\right.\right. \\
& -\breve{W}_{2 j}\left(\omega_{1} v_{2}-\omega_{2} v_{1}\right)+\breve{Y}_{4 j}\left(\omega_{3}^{2}-\omega_{2}^{2}\right)+\breve{Y}_{5 j} \omega_{1} \omega_{2} \\
& \left.\left.+\breve{Z}_{4 j}\left(\omega_{2}^{2}-\omega_{3}^{2}\right)+\check{Z}_{6 j} \omega_{1} \omega_{3}\right] q_{j}+2\left[\breve{Y}_{5 j} \omega_{3}-\breve{Z}_{6 j} \omega_{2}\right] \dot{q}_{j}\right\}
\end{aligned}
$$




$$
\begin{aligned}
& \bar{F}_{2}=m_{A}\left\{d_{1}\left[\omega_{1}\left(v_{2}+\omega_{3} d_{1}-\omega_{1} d_{3}\right)-\omega_{2}\left(v_{1}+\omega_{2} d_{3}-\omega_{3} d_{2}\right)\right]\right. \\
& \left.+d_{3}\left[\omega_{3}\left(v_{2}+\omega_{3} d_{1}-\omega_{1} d_{3}\right)-\omega_{2}\left(v_{3}+\omega_{1} d_{2}-\omega_{2} d_{1}\right)\right]\right\} \\
& +\omega_{3} \omega_{1}\left(J_{33}-J_{11}\right)-\omega_{2} \omega_{3} J_{12}-\omega_{3}^{2} J_{13}+\omega_{1}^{2} J_{31} \\
& +\omega_{1} \omega_{2} J_{32}-I_{B 2} \omega_{3} \omega_{1}+P_{1}\left(\omega_{1} v_{2}-\omega_{2} v_{1}\right)+P_{2} \omega_{1} \omega_{3} \\
& +\sum_{j=1}^{v}\left\{\left[\breve{W}_{1}\left(\omega_{1} v_{2}-\omega_{2} v_{1}\right)+\breve{W}_{3 j}\left(\omega_{3} v_{2}-\omega_{2} v_{3}\right)\right.\right. \\
& +2 \breve{X}_{1 j} \omega_{1} \omega_{3}^{\prime}+\breve{X}_{2 j} \omega_{2} \omega_{3}+\breve{X}_{3 j}\left(\omega_{3}^{2}-\omega_{1}^{2}\right)+\breve{Y}_{4 j} \omega_{1} \omega_{2} \\
& \left.+\breve{Y}_{5 j}\left(\omega_{3}^{2}-\omega_{1}^{2}\right)-\breve{Z}_{4 j} \omega_{1} \omega_{2}-\breve{Z}_{6 j} \omega_{2} \omega_{3}\right] q_{j} \\
& \left.+2\left[\breve{X}_{2 j} \omega_{1}-\breve{X}_{1 j} \omega_{2}-\breve{Y}_{4 j} \omega_{3}\right] \dot{q}_{j}\right\} \\
& \bar{F}_{3}=m_{A}\left\{d_{2}\left[\omega_{2}\left(v_{3}+\omega_{1} d_{2}-\omega_{2} d_{1}\right)-\omega_{3}\left(v_{2}+\omega_{3} d_{1}-\omega_{1} d_{3}\right)\right]\right. \\
& \left.+d_{1}\left[\omega_{1}\left(v_{3}+\omega_{1} d_{2}-\omega_{2} d_{1}\right)-\omega_{3}\left(v_{1}+\omega_{2} d_{3}-\omega_{3} d_{2}\right)\right]\right\} \\
& +\omega_{1} \omega_{2}\left(J_{11}-J_{22}\right)+\omega_{2}^{2} J_{12}+\omega_{2} \omega_{3} J_{13}-\omega_{1}^{2} J_{21} \\
& -\omega_{1} \omega_{3} J_{23}+I_{B 3} \omega_{1} \omega_{2}+P_{1}\left(\omega_{1} v_{3}-\omega_{3} v_{1}\right)-P_{2} \omega_{1} \omega_{2} \\
& +\sum_{j=1}^{v}\left\{\left[\breve{W}_{1 j}\left(\omega_{1} v_{3}-\omega_{3} v_{1}\right)+\breve{W}_{2 j}\left(\omega_{2} v_{3}-\omega_{3} v_{2}\right)-2 \breve{X}_{1 j} \omega_{1} \omega_{2}\right.\right. \\
& +\breve{X}_{2 j}\left(\omega_{1}^{2}-\omega_{2}^{2}\right)-\breve{X}_{3 j} \omega_{2} \omega_{3}-\breve{Y}_{4 j} \omega_{1} \omega_{3}-\breve{Y}_{5 j} \omega_{2} \omega_{3}+\breve{Z}_{4 j} \omega_{1} \omega_{3} \\
& \left.\left.+\breve{Z}_{6 j}\left(\omega_{2}^{2}-\omega_{1}^{2}\right)\right] q_{j}+2\left[-\breve{X}_{1 j} \omega_{3}+\breve{X}_{3 j} \omega_{1}+\breve{Z}_{4 j} \omega_{2}\right] \dot{q}_{j}\right\} \\
& \bar{F}_{4}=m_{A}\left\{\omega_{3}\left(v_{2}+\omega_{3} d_{1}-\omega_{1} d_{3}\right)-\omega_{2}\left(v_{3}+\omega_{1} d_{2}-\omega_{2} d_{1}\right)\right\} \\
& +m_{B}\left\{\omega_{3} v_{2}-\omega_{2} v_{3}\right\}+P_{1}\left(\omega_{2}^{2}+\omega_{3}^{2}\right) \\
& +\sum_{j=1}^{\nu}\left\{\left[\breve{W}_{1}\left(\omega_{2}^{2}+\omega_{3}^{2}\right)-\breve{W}_{2 j} \omega_{1} \omega_{2}-\breve{W}_{3 j} \omega_{1} \omega_{3}\right] q_{j}\right. \\
& \left.+2\left[\breve{W}_{2 j} \omega_{3}-\breve{W}_{3 j} \omega_{2}\right] \dot{q}_{j}\right\} \\
& \bar{F}_{5}=m_{A}\left\{\omega_{1}\left(v_{3}+\omega_{1} d_{2}-\omega_{2} d_{1}\right)-\omega_{3}\left(v_{1}+\omega_{2} d_{3}-\omega_{3} d_{2}\right)\right\} \\
& +m_{B}\left\{\left(\omega_{1} v_{3}-\omega_{3} v_{1}\right)\right\}-P_{1} \omega_{1} \omega_{2} \\
& +\sum_{j=1}^{\nu}\left\{\left[-\breve{W}_{1 j} \omega_{1} \omega_{2}+\breve{W}_{2 j}\left(\omega_{1}^{2}+\omega_{3}^{2}\right)-\breve{W}_{3 j} \omega_{2} \omega_{3}\right] q_{j}\right. \\
& \left.+2\left[-\breve{W}_{1 j} \omega_{3}+\breve{W}_{3 j} \omega_{1}\right] \dot{q}_{j}\right\}
\end{aligned}
$$

$$
{ }^{N} C^{A}=\left[\begin{array}{c}
c_{v+5} c_{v+6} \\
s_{v+4} s_{v+5} c_{v+6}+s_{v+6} c_{v+4} \\
-c_{v+4} s_{v+5} c_{v+6}+s_{v+6} s_{v+4}
\end{array}\right.
$$

$$
\begin{aligned}
\bar{F}_{6} & =m_{A}\left\{\omega_{2}\left(v_{1}+\omega_{2} d_{3}-\omega_{3} d_{2}\right)-\omega_{1}\left(v_{2}+\omega_{3} d_{1}-\omega_{1} d_{3}\right)\right\} \\
& +m_{B}\left\{\omega_{2} v_{1}-\omega_{1} v_{2}\right\}-P_{1} \omega_{1} \omega_{3} \\
& +\sum_{j=1}^{v}\left\{\left[-\breve{W}_{1 j} \omega_{1} \omega_{3}-\breve{W}_{2 j} \omega_{2} \omega_{3}+\breve{W}_{3 j}\left(\omega_{1}^{2}+\omega_{2}^{2}\right)\right] q_{j}\right. \\
& \left.+2\left[\breve{W}_{1 j} \omega_{2}-\breve{W}_{2 j} \omega_{1}\right] \dot{q}_{j}\right\}
\end{aligned}
$$

The matrices $\mathbf{K}^{*}$ and $\breve{\mathbf{F}}$ appearing in Eq. (22) serve merely to extract terms from $\mathbf{K}$ and $\mathbf{F}$, which are explicit functions of $\dot{u}_{v+i}^{*}(i=1, \ldots, 6)$. Hence, even though $\mathbf{K}^{*}$ and $\breve{\mathbf{F}}$ contain $\dot{u}_{v+i}^{*}$ $(i=1, \ldots, 6)$, the elements $\mathscr{K}_{i j}$ and $\mathscr{F}_{i}$ of matrices $\mathscr{K}$ and $\mathscr{F}$, respectively, do not contain such terms.

It is clear from Eq. (21) that the matrix $\mathscr{M}$ is nonsymmetric. This unfortunate fact is a consequence of linearizing in some, but not all, of the generalized coordinates and generalized speeds. One may, however, employ a symmetric version of $\mathscr{M}$ for purposes of computational efficiency. To do this, the term
$\left(\mathbf{M}^{*}\right)^{T}$ is added to the lower left partition of $\mathscr{M}$ in Eq. (21). This does not affect the accuracy of the solution since each term in $\mathbf{M}^{*}$ is a function of $q_{i}(i=1, \ldots, v)$; thus, when $q_{j}$ is multiplied by $\mathbf{M}_{j i}^{*}(i=1, \ldots, 6 ; j=1, \ldots v)$, negligible terms of degree two and higher in $q_{1}, \ldots, q_{v}, \dot{q}_{1}, \ldots, \dot{q}_{v}$ result. Another, less rigorous, method to symmetrize the mass matrix is simply to discard the terms arising from $\mathbf{M}^{*}$. Although no formal analytical justification for this action has yet been developed, an extensive study of simulations with and without these terms suggest that their influence is indeed negligible for practical applications.

\section{Kinematical Equations}

In addition to the $v+6$ first-order dynamical equations governing the motion of $A$ and $B$ in $N$, it is necessary to form at least $v+6$ first-order kinematical equations before a complete solution for the position and orientation of $A$ and $B$ in $N$ can be obtained. The first $v$ kinematical equations are

$$
\dot{q}_{i(\overline{\overline{6}})} u_{i}^{*} \quad(i=1, \ldots, v)
$$

To write the remaining six equations, one must introduce six additional generalized coordinates, $q_{v+1}, \ldots, q_{v+6}$, which can be defined in many ways. Here they will be chosen to describe the position of point $O$ and the inertial orientation of $A$. Accordingly, let

$$
q_{v+i} \triangleq p^{80} \cdot n_{i} \quad(i=1,2,3)
$$

where $p^{\mathscr{E O}}$ is the position vector from a particular point $\mathscr{E}$, fixed in $N$, to point $O$, and $n_{1}, n_{2}, n_{3}$, form a dextral set of mutually perpendicular unit vectors fixed in $N$. To describe the orientation of $A$ in $N$, align $a_{1}, a_{2}, a_{3}$ with $n_{1}, n_{2}, n_{3}$, respectively, and subject $A$ to successive dextral rotations in $N$ of amounts $q_{v+4}, q_{v+5}$, and $q_{v+6}$ about lines parallel to $a_{1}, a_{2}$, and $a_{3}$, respectively. After such a reorientation, the unit vectors $a_{i}$ and $n_{j}(i, j=1,2,3)$ are related by a direction cosine matrix ${ }^{N} C^{A}$ in the following way:

$$
\left[\begin{array}{lll}
a_{1} & a_{2} & a_{3}
\end{array}\right]=\left[\begin{array}{lll}
n_{1} & n_{2} & n_{3}
\end{array}\right]^{N} C^{A}
$$

with ${ }^{N} C^{A}$ given by

$$
\left.\begin{array}{cc}
-c_{v+5} s_{v+6} & s_{v+5} \\
-s_{v+4} s_{v+5} s_{v+6}+c_{v+6} c_{v+4} & -s_{v+4} c_{v+5} \\
c_{v+4} s_{v+5} s_{v+6}+c_{v+6} s_{v+4} & c_{v+4} c_{v+5}
\end{array}\right]
$$

With the position of point $O$ and the orientation of $A$ described in terms of generalized coordinates as stated, the remaining six kinematical equations are given as

$$
\begin{aligned}
& \dot{q}_{v+1}=v_{1}^{N} C_{11}^{A}+v_{2}{ }^{N} C_{12}^{A}+v_{3}{ }^{N} C_{13}^{A} \\
& \dot{q}_{v+2}=v_{1}^{N} C_{21}^{A}+v_{2}^{N} C_{22}^{A}+v_{3}{ }^{N} C_{23}^{A} \\
& \dot{q}_{v+3}=v_{1}^{N} C_{31}^{A}+v_{2}{ }^{N} C_{32}^{A}+v_{3}{ }^{N} C_{33}^{A} \\
& \dot{q}_{v+4}=\left(\omega_{1} c_{v+6}-\omega_{2} s_{v+6}\right) / c_{v+5} \\
& \dot{q}_{v+5}=\omega_{1} s_{v+6}+\omega_{2} c_{v+6} \\
& \dot{q}_{v+6}=\left(-\omega_{1} c_{v+6}+\omega_{2} s_{v+6}\right) s_{v+5} / c_{v+5}+\omega_{3}
\end{aligned}
$$

Here ${ }^{N} C_{i j}^{A}$ represents the element in the $i$ th row and $j$ th column of ${ }^{N} C^{A}$. Because a numerical solution of Eqs. (63) and (65) would become unbounded when $\cos q_{v+5}=0$ (e.g., $q_{v+5}=90 \mathrm{deg}$ ), it may be expedient to replace the three 
kinematical Eqs. (63-65) with a set of four numerically well-behaved equations involving quantities $\varepsilon_{1}, \varepsilon_{2}, \varepsilon_{3}$, and $\varepsilon_{4}$, known as Euler parameters. These are related to the direction cosines ${ }^{N} C_{i j}^{A}$ in such a way that ${ }^{9}$

$$
{ }^{N} C^{A}=\left[\begin{array}{lll}
1-2 \varepsilon_{2}^{2}-2 \varepsilon_{3}^{2} & 2\left(\varepsilon_{1} \varepsilon_{2}-\varepsilon_{3} \varepsilon_{4}\right) & 2\left(\varepsilon_{3} \varepsilon_{1}+\varepsilon_{2} \varepsilon_{4}\right) \\
2\left(\varepsilon_{1} \varepsilon_{2}+\varepsilon_{3} \varepsilon_{4}\right) & 1-2 \varepsilon_{3}^{2}-2 \varepsilon_{1}^{2} & 2\left(\varepsilon_{2} \varepsilon_{3}-\varepsilon_{1} \varepsilon_{4}\right) \\
2\left(\varepsilon_{3} \varepsilon_{1}-\varepsilon_{2} \varepsilon_{4}\right) & 2\left(\varepsilon_{2} \varepsilon_{3}+\varepsilon_{1} \varepsilon_{4}\right) & 1-2 \varepsilon_{1}^{2}-2 \varepsilon_{2}^{2}
\end{array}\right]
$$

Kinematical equations formed in terms of the Euler parameters appear as ${ }^{9}$

$$
\begin{array}{cc}
\dot{\varepsilon}_{1}=\frac{1}{2}\left(\omega_{1} \varepsilon_{4}-\omega_{2} \varepsilon_{3}+\omega_{3} \varepsilon_{2}\right), & \dot{\varepsilon}_{2}=\frac{1}{2}\left(\omega_{1} \varepsilon_{3}+\omega_{2} \varepsilon_{4}-\omega_{3} \varepsilon_{1}\right) \\
\dot{\varepsilon}_{3}=\frac{1}{2}\left(-\omega_{1} \varepsilon_{2}+\omega_{2} \varepsilon_{1}+\omega_{3} \varepsilon_{4}\right), & \dot{\varepsilon}_{4}=-\frac{1}{2}\left(\omega_{1} \varepsilon_{1}+\omega_{2} \varepsilon_{2}+\omega_{3} \varepsilon_{3}\right)
\end{array}
$$

Consequently, Eqs. (60-62) and (67) and (68) can be used effectively in a numerical simulation in order to produce information concerning the position and orientation of the base at any instant.

\section{External Forces}

In order to perform simulations of practical interest in connection with flexible spacecraft and satellites, the equations of motion presented here should be augmented with additional contributions to the generalized active forces to account for realistic external forces such as gravity, active control, atmospheric drag, solar heating, etc. The procedure for including the contribution of a very general external forcing function in the system generalized active forces was presented in Eq. (18), where the terms $(R)^{A^{*}},(R)_{x=\sigma}^{C} ;(R)^{C}$, and $(T)^{A},(T)_{x=\sigma}^{\mathrm{d} B},(T)^{\mathrm{d} B}$, defined previously, can be assumed to represent external force effects. Any set of forces can be expressed in terms of these quantities or can be included directly in the system generalized active forces by simply dot-multiplying each force with the partial velocity, in $N$, of

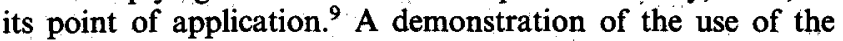
procedure in accounting for active control laws and gravitational effects will be given here. The effects of solar-induced heating and atmospheric drag have been investigated for similar systems in Refs. 10-12, and will not be considered further.

To develop the expressions for the contribution of gravitational forces and control forces to the generalized active forces for a system consisting of one beam attached to a rigid base, proceed as follows. Assume the function of an active control system is to apply a set of time-dependent forces to the system, this set being equivalent to a couple of torque $\left(T_{\text {cont }}\right)^{A}$ acting on $A$ and a force $\left(F_{\text {cont }}\right)^{\tau}$ applied at a point $\tau$ located on the elastic axis of $B$ at the tip. Then the control system contribution to the generalized active forces $F_{i}(i=1, \ldots, v+6)$ is

$$
\left(F_{i}\right)_{\mathrm{cont}}={ }^{N} \omega_{i}^{A} \cdot\left(T_{\text {cont }}\right)^{A}+{ }^{N} \tilde{v}_{i}^{\tau} \cdot\left(F_{\text {cont }}\right)^{\tau} \quad(i=1, \ldots, v+6)
$$

where ${ }^{N} \tilde{\boldsymbol{v}}_{i}^{\tau}$ is the $i$ th linearized partial velocity of $\tau$ in $N$. With $\left(\boldsymbol{T}_{\text {cont }}\right)^{A}$ and $\left(\boldsymbol{F}_{\text {cont }}\right)^{\tau}$ expressed in component form as

$$
\begin{aligned}
& \left(T_{\text {cont }}\right)^{A}=T_{1 c} a_{1}+T_{2 c} a_{2}+T_{3 c} a_{3} \\
& \left(F_{\text {cont }}\right)^{\tau}=F_{1 c} a_{1}+F_{2 c} a_{2}+F_{3 c} a_{3}
\end{aligned}
$$

the terms $\left(F_{i}\right)_{\text {cont }}(i=1, \ldots, v+6)$ appear explicitly as

$$
\begin{aligned}
& \left(F_{i}\right)_{\mathrm{cont}}=\left\{\phi_{1 i}(L)-\sum_{j=1}^{v}\left[\beta_{i j}(L)+\gamma_{i j}(L)\right] q_{j}\right\} F_{1 c} \\
& +\left\{\phi_{2 i}(L)\right\} F_{2 c}+\left\{\phi_{3 i}(L)\right\} F_{3 c} \quad(i=1, \ldots, v) \\
& \left(F_{v+1}\right)_{\mathrm{cont}}=T_{1 c}-\sum_{j=1}^{v} \phi_{3 j}(L) q_{j} F_{2 c}+\sum_{j=1}^{v} \phi_{2 j}(L) q_{j} F_{3 c} \\
& \left(F_{v+2}\right)_{\mathrm{cont}}=T_{2 c}+\sum_{j=1}^{v} \phi_{3 j}(L) q_{j} F_{1 c}-\left[L+\sum_{j=1}^{v} \phi_{1 j}(L) q_{j}\right] F_{3 c} \\
& \left(F_{v+3}\right)_{\mathrm{cont}}=T_{3 c}-\sum_{j=1}^{v} \phi_{2 j}(L) q_{j} F_{1 c}+\left[L+\sum_{j=1}^{v} \phi_{1 j}(L) q_{j}\right] F_{2 c} \\
& \left(F_{v+4}\right)_{\mathrm{cont}}=F_{1 c} \quad\left(F_{v+5}\right)_{\mathrm{cont}}=F_{2 c} \quad\left(F_{v+6}\right)_{\mathrm{cont}}=F_{3 c}
\end{aligned}
$$

where $\beta_{i j}$ and $\gamma_{i j}$ are modal integrals defined in Ref. 1 to account for spin-induced dynamic stiffness effects.

Next, consider the gravitational forces exerted on $A$ and $B$ by a particle of mass $\bar{m}$ located at a point $\mathscr{E}$ fixed in a Newtonian reference frame. The contribution of these forces to the generalized active forces can be approximated as

$$
\begin{aligned}
& \left(F_{i}\right)_{\mathrm{grav}}={ }^{N} \boldsymbol{v}_{i}^{A^{*}} \cdot\left\{-G \bar{m} m_{A} p^{\delta A^{*}}\left[\left(\boldsymbol{p}^{\delta A^{*}}\right)^{2}\right]^{-3 / 2}\right\} \\
& +{ }^{N} \omega_{i}^{A}:\left\{3 G \bar{m}\left[\left(p^{8 A^{*}}\right)^{2}\right]^{-5 / 2}\left[p^{\& A^{*}} \times \underline{\mathbf{J}} \cdot \boldsymbol{p}^{8 A^{*}}\right]\right\} \\
& +\int_{0}^{L}{ }^{N_{\tilde{v}} C} \cdot\left\{-G \bar{m} \rho p^{\& C}\left[\left(\boldsymbol{p}^{\& C}\right)^{2}\right]^{-3 / 2}\right\} \mathrm{d} x \quad(i=1, \ldots, v+6)
\end{aligned}
$$

where $p^{\mathscr{E} A^{*}}$ and $p^{\mathscr{E} C}$ are position vectors from point $\mathscr{E}$ to points $A^{*}$ and $C$, respectively.

\section{Simulation Results}

A computational algorithm based on the previously presented equations has been developed and used to simulate the free-flying motion of actively controlled spacecraft during various practical maneuvers. Some results from these simulations will be shown here to 1) illustrate the use of the theory incorporating free base motion and external forces and 2) point out differences between this theory and conventional multibody methods.

Two orbital motions will be considered: one concerning a controlled planar motion in low Earth orbit and the other involving a large-angle, three-dimensional reorientation maneuver followed by a precise pointing motion during geosynchronous orbit. Before these are discussed, however, a few simulation results will be presented for motions in which gravity is neglected entirely; these focus attention primarily on flexible-body and rigid-body interaction.

\section{Rigid-Body/Flexible-Body Interaction}

Many satellites used to conduct scientific experiments and perform measurements of magnetic properties, electrical properties, radio-wave transmission, etc., in space, consist of a fairly rigid hub and one or more flexible booms extending from the hub and having instrumentation packages attached. In order to measure properties of the surrounding plasma, a number of maneuvers are executed, ${ }^{13}$ such as spin-up, spindown, and steady spin about principal axes of the base, slews about principal base axes, and orbit change maneuvers. Spinup is often accomplished by firing short bursts of gas from thrusters attached to the base. To simulate such a procedure, consider the system depicted in Fig. 2 to be at rest initially with no external forces applied. Starting with $t=0$, a 5-s pulse of a set of thrusters is represented by letting

$$
F_{1 c}=F_{2 c}=F_{3 c}=T_{1 c}=T_{2 c}=0
$$




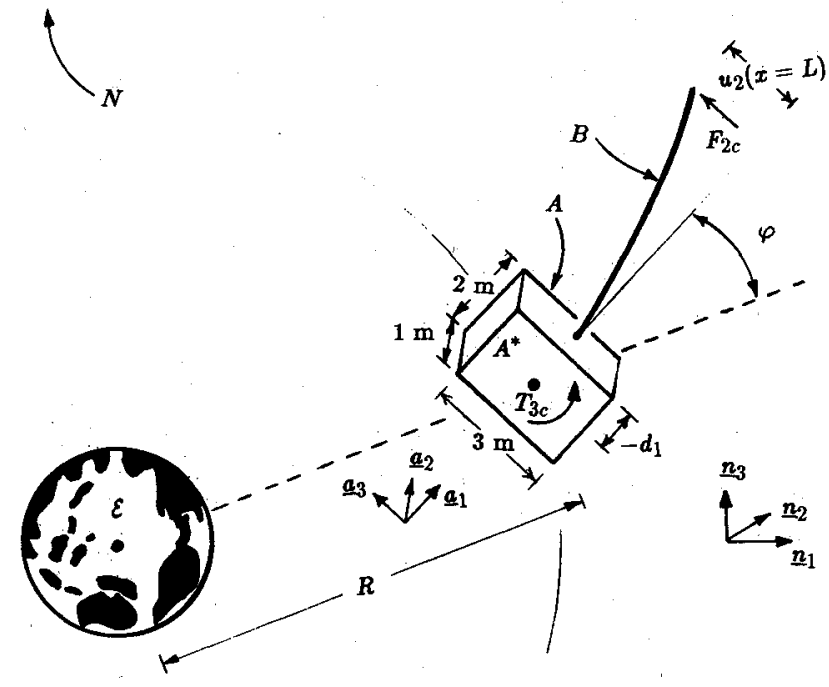

Fig. 2 Satelite system.

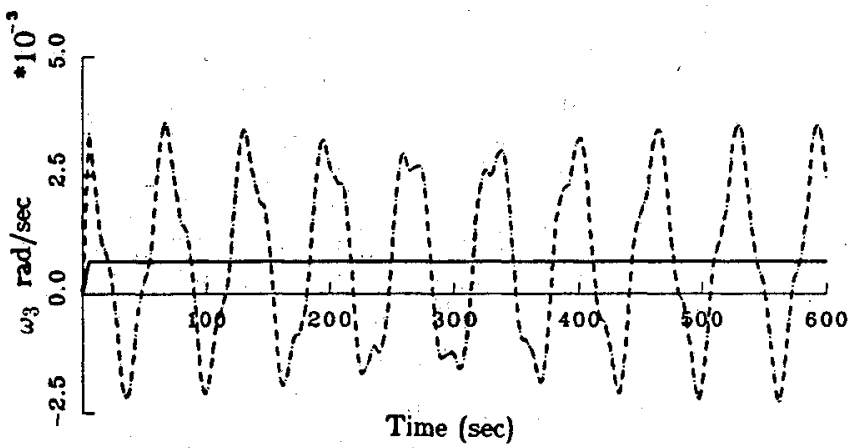

Fig. 3 Single-pulse spin-up: rotational speed.

$$
T_{3 c}= \begin{cases}0.1 \mathrm{~N} \cdot \mathrm{m} & 0<t \leq 5 \mathrm{~s} \\ 0.0 \mathrm{~N} \cdot \mathrm{m} & 5<t \leq 600 \mathrm{~s}\end{cases}
$$

where $F_{1 c}, F_{2 c}, F_{3 c}, T_{1 c}, T_{2 c}$, and $T_{3 c}$ are the terms introduced at the end of the last section to account for control system actuation. The free-flying base $A$ is a solid, $120-\mathrm{kg}$, rectangular parallelepiped with sides of length 2,3 , and $1 \mathrm{~m}$, parallel to $a_{1}, a_{2}, a_{3}$, respectively. The flexible appendage $B$ is a uniform cantilever beam of length $20-\mathrm{m}$, flexural rigidities $E_{o} I_{2}=E_{o} I_{3}=5 \mathrm{~N} \cdot \mathrm{m}^{2}$, torsional rigidity $G \kappa^{\prime}=6 \mathrm{~N} \cdot \mathrm{m}^{2}$, cross-sectional area $A_{o}=4 \times 10^{-4} \mathrm{~m}^{2}$, and a mass per unit length $\rho=0.2 \mathrm{~kg} / \mathrm{m}$.

If the beam appendage were rigid, the time history of the angular rate $\dot{\omega}_{3}$ associated with the based-fixed unit vector $a_{3}$ would appear as shown by the solid line in Fig. 3, where $\omega_{3}$ is plotted vs time for $600 \mathrm{~s}$. Soon after the 5-s pulse, $\omega_{3}$ reaches a steady-state value, and spin-up is complete. However, when the flexibility of the appendage is taken into account in the simulation, one obtains the dashed line result for $\omega_{3}$ in Fig. 3. These results clearly show the effect that motion of the flexible appendage (see Fig. 4) has on motion of the base: Instead of a constant-velocity steady-state spin, the hub alternately speeds up and slows down in a pulsating fashion. This rigid-body/elastic-body coupling, which plays such an important role here, is not accurately treated in the conventional multibody programs discussed in Refs. 2-6 unless the fiexible appendage is discretized or substructured in the multibody system model. Suppose that the previous simulation is repeated with $T_{3 c}$ changed to $1.0 \mathrm{~N} \cdot \mathrm{m}$. Using standard appendage modes and a continuous, unsubstructured beam model along with an algorithm based on the conventional modal modeling approach used in these programs, one

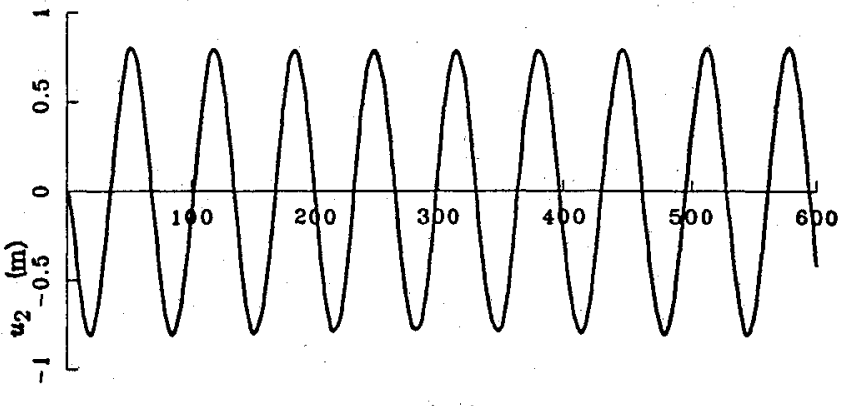

Time (sec)

Fig. 4 Single-pulse spin-up: beam transverse tip deflection.

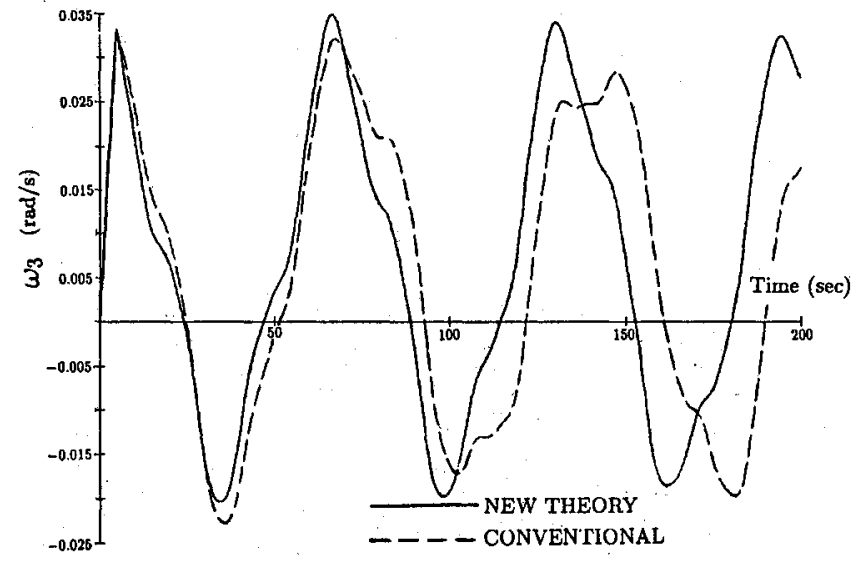

Fig. 5 Single-pulse spin-up: comparison of theories.

obtains the dashed-line curve for $\omega_{3}$ vs time shown in Fig. 5 for the first $200 \mathrm{~s}$ of motion; the solid line represents results obtained with an algorithm based on the theory in this paper combined with that of Ref. 1 .

A number of techniques have been proposed for controlling the amplitude of the in-plane, beam-tip deflection, shown in Fig. 4, and thereby reducing the steady-state variation in $\omega_{3}$. One such strategy, termed the "half-period-pulsing" method, ${ }^{13}$ involves firing the thrusters twice in a precise sequence. The initial firing imparts a certain angular momentum to the system resulting in both rotation of the base and vibration of the appendage. Then, a second thruster pulse of equal magnitude and direction to the first is applied after a time interval equal to one-half the period of the fundamental bending mode of the system. [The term fundamental bending mode, as used here, is to be interpreted as the first mode obtained by solving an eigenproblem resulting from 1) linearizing the previous $v+6$ nonlinear equations about a state of constant-speed $\left(\omega_{3}\right)$ rotational motion and zero deformation and 2) assuming an exponential solution.] The idea is to use the second pulse both to increase spin and negate vibrational momentum. This strategy is investigated here in order to illustrate the effectiveness of the current theory and accompanying algorithm in treating controlled spacecraft motion. Figure 6a shows the time histories of $\omega_{3}$ and the in-plane tip deflection $u_{2}(x=L)$ obtained from a simulation identical to the single-pulse simulation except that a second pulse is fired at a time ( $33 \mathrm{~s}$ ) equal to approximately half the period of the fundamental mode calculated for a case of zero steady-state spin. After the second pulse, the tip deflection is indeed reduced considerably, and the mean spin rate is increased. This strategy appears to be effective and can be repeated indefinitely to achieve any desired spin rate. Figure $6 \mathrm{~b}$ displays results from a simulation during which double pulses of 5-s duration and 33-s separation are repeated for the first $300 \mathrm{~s}$ of the motion. 
a) Double pulse spin-up

b) Nine pulse spin-up

Fig. 6 Multipulse spin-up maneuvers.

Fig. 7 Uncontrolled librational motion of flexible satellite.
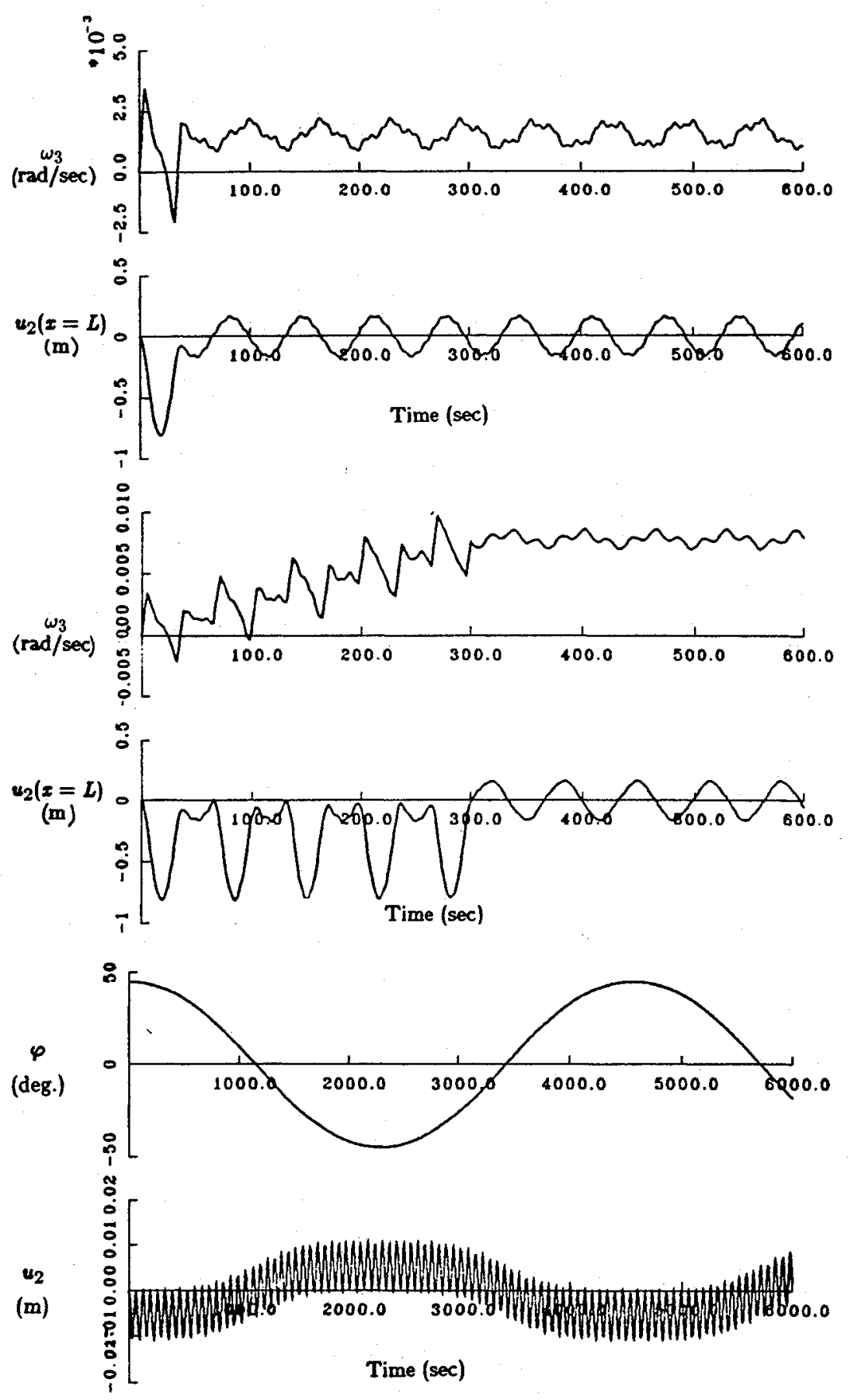

To use this strategy in an open-loop fashion requires an accurate model to provide an indication of the frequency of the fundamental mode as the spin speed increases. Conventional methods indicate that this frequence decreases with increasing spin speed whereas, in reality, the frequency of the fundamental mode increases. For the stimulation reported in Fig. 5, the error in the fundamental frequency found using the conventional modal modeling theory was approximately $10 \%$. Development of optimal control schemes based on such an erroneous model could lead to dynamic instabilities unless sufficient measurement capabilities and control law robustness were built into the system.

\section{Planar Librational Motion}

Next, the effect of appendage flexibility on the behavior of a satellite during orbit will be investigated using the same system introduced earlier. First a planar librational motion, studied previously in Ref. 9 (p. 330), will be considered followed by a three-dimensional, large-angle reorientation of an orbiting satellite.
For the first motion, consider the mass center $A^{*}$ of $A$ to be initially placed at a point $622 \mathrm{~km}$ above the Earth's surface with a velocity in $N$ (Newtonian reference frame) equal to the velocity of a particle that occupies the same position as $A^{*}$ and is moving on a circular orbit around the Earth. The base $A$ is given an initial angular velocity such that it is not rotating relative to a line joining $\mathscr{E}$ (Earth's mass center) and $A^{*}$, and the beam appendage is initially at rest in $A$. Furthermore, the base-fixed unit vector $a_{3}$ is aligned with the orbit normal, and the angle $\varphi$, shown in Fig. 2, is given an initial value of $45 \mathrm{deg}$. If no active control is applied to the system subsequent to the initial time, simulation results for the angle $\varphi$ and the beam-tip transverse deflection $u_{2}$ (see Fig. 7) reveal that the system oscillates about the local vertical and the beam vibrates.

Suppose, however, that it is desired to control the satellite actively in such a way as to cause $A^{*}$ to move on a circular orbit with $\varphi=0$. The system then moves in the so-called spoke mode, with the beam's elastic axis perfectly aligned with the local vertical at all times. If the beam appendage $B$ is 
considered to be rigid, a control scheme to accomplish this objective can be designed quite easily. For example, one can set $F_{1 c}=F_{2 c}=F_{3 c}=T_{1 c}=T_{2 c}=0$ for all time and assign $T_{3 c}$ (the $a_{3}$ measure number of the torque applied to $A$ ) to be a linear function of two quantities $\Omega$ and $V$, referred to as the attitude rate and tip speed, respectively. These quantities are defined as

$$
\Omega \triangleq \dot{\varphi}, \quad V \triangleq\left(L-d_{1}\right) \dot{\varphi}+\sum_{j=1}^{\nu}\left[\phi_{1} q_{j} \dot{\varphi}+\phi_{2 j} \dot{q}_{j}\right]
$$

where the summations are simply neglected for the case when $B$ is considered to be rigid. Thus, $T_{3 c}$ can be written in the form

$$
T_{3 c}=-\left(k_{\Omega} \Omega+k_{V} V\right)
$$

where $k_{\Omega}$ and $k_{V}$ are proportional control gains. Suppose that $k_{V}$ is zero and $k_{\Omega}$ is chosen so as to provide an underdamped response of the satellite when the appendage is considered to be rigid. With $k_{\Omega}=1.185 \mathrm{~N} \cdot \mathrm{m} \cdot \mathrm{s}$ and initial conditions identical to those used to produce Fig. 7, one obtains the simulation results shown in Fig. 8a, where the dashed curves with black dots represent the appropriate underdamped response for the case in which the appendage is considered as rigid, whereas the solid curves represent the response of the system when $B$ is characterized by three transverse vibrational bending modes in the $a_{1}-a_{2}$ plane. These results suggest that the control system, designed as if $B$ were rigid, works reasonably well even when $B$ is significantly deformable. Although the beam does vibrate, the angle $\varphi$ is reduced to a very small value, with minimal control effort beyond that required for a rigid appendage.

One can, of course, also use this theory and associated algorithms to investigate the usefulness of various modal control schemes, as well as study the possible deleterious effects of noncolocated control schemes. For example, assume that the scalar $T_{3 c}$ that was previously made proportional to the attitude rate of the base is now assigned to be proportional to the speed $V$ measured at the beam tip. Thus, measurements are made at the tip location, and control torques are applied at the base. Choosing $k_{V}$ in such a way $\left(k_{V}=0.0575 \mathrm{~N} \cdot \mathrm{s}\right)$ so as to yield an underdamped response of the system when $B$ is rigid and performing a second simulation with identical initial conditions and parameters as those used in Fig. 8a, one obtains the results illustrated in Fig. 8b. These results substantiate the well-known problems with employing noncolocated control (sensors and actuators at separate locations) for flexible spacecraft. Unless a more robust controller than the one employed here is used, instability can be expected.

\section{Large-Angle Reorientation Maneuvers}

Last, a pointing maneuver of importance for both communication and strategic defense satellites will be studied. Consider the system shown in Fig. 2 to be moving in a nearly circular, equatorial, geosynchronous orbit, with the beam's elastic axis pointing directly inward toward the center of the Earth. Suppose, furthermore, that at some specific time, it is desired to reorient the satellite and change its target. Such a maneuver could be accomplished by firing different sets of base-fixed thrusters in sequential fashion with the intent of causing the base to rotate successively about lines parallel to base principal axes. With a proper thruster firing sequence, any desired orientation could, in theory, be achieved. Using this notion, the following control strategy has been formulated by considering $B$ to be rigid, as was done in the preceding examples. If $\mathscr{J}_{1}, \mathscr{J}_{2}$, and $\mathscr{J}_{3}$ denote central principal moments of inertia of the "rigid" satellite corresponding to principal axes parallel, respectively, to $a_{1}, a_{2}$, and $a_{3}$, then attitude behavior of the rigid satellite is governed by Euler's dynamical equations written in the form

$$
\mathscr{J}_{1} \dot{\omega}_{1}-\left(\mathscr{J}_{2}-\mathscr{J}_{3}\right) \omega_{2} \omega_{3}=T_{1}+G_{1}
$$
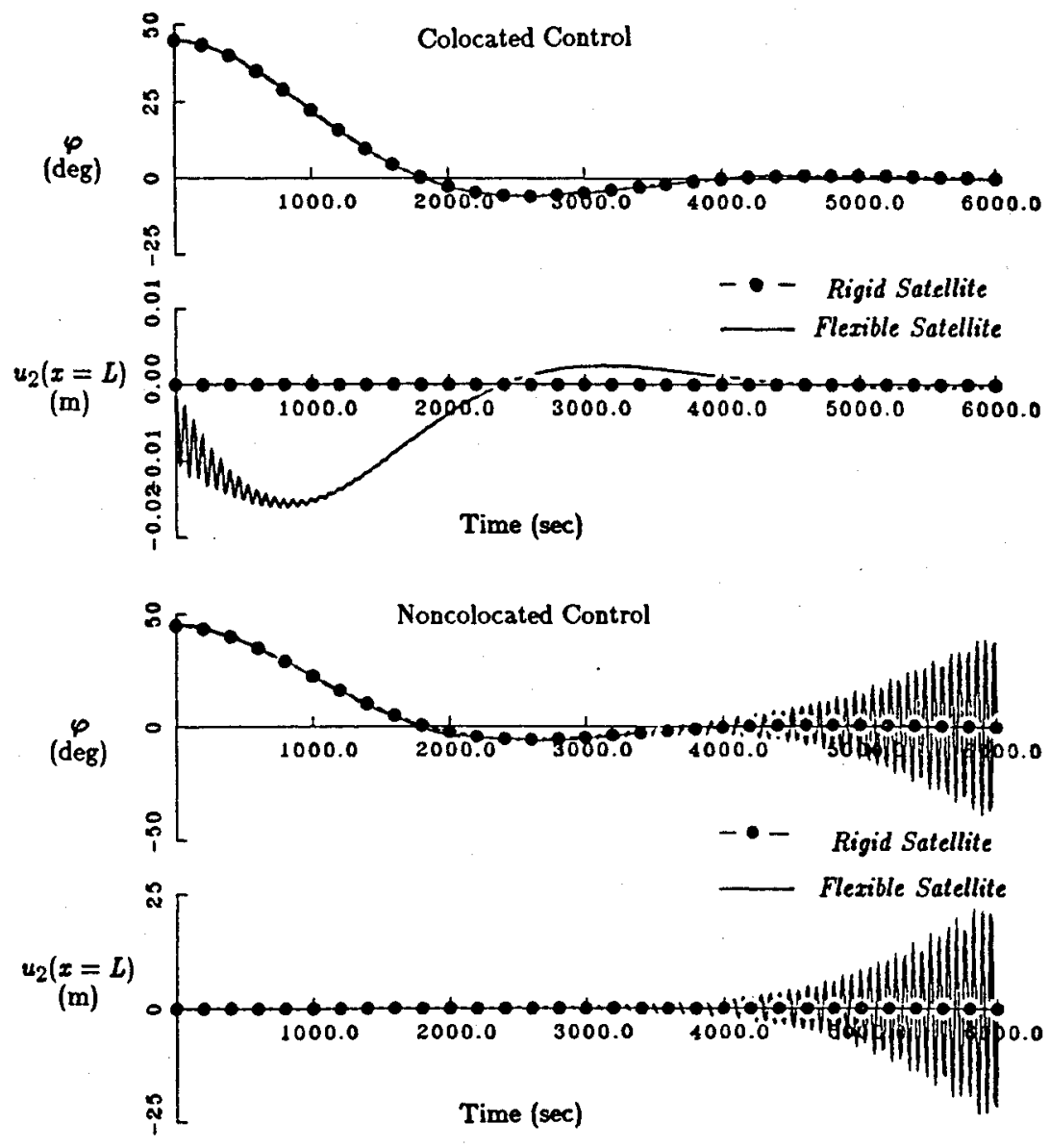

a) Stable librational motion

b) Unstable librational motion

Fig. 8 Controlled planar librational motion. 


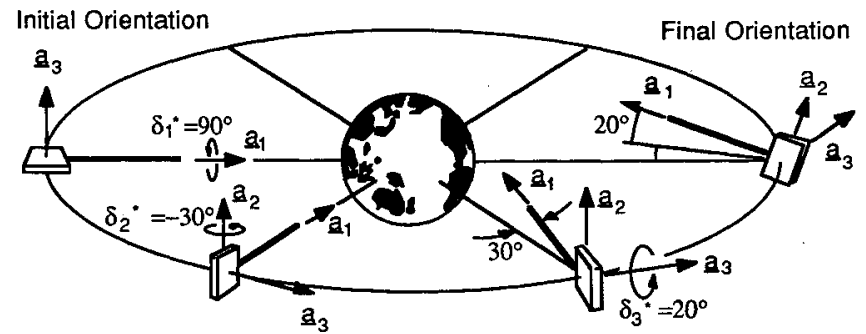

Fig. 9 Reorientation of a satellite in a geosynchronous orbit.

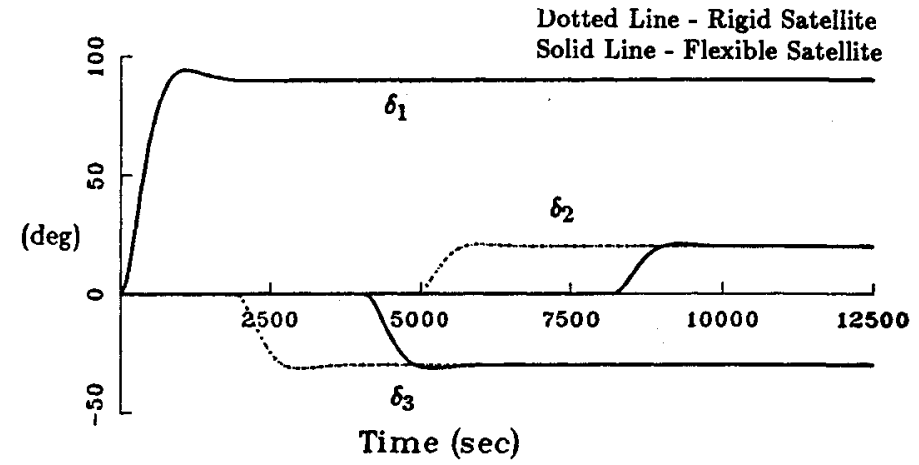

Fig. 10 Controlled reorientation-rotation angles.
Fig. 11 Beam deformation during reorientation.
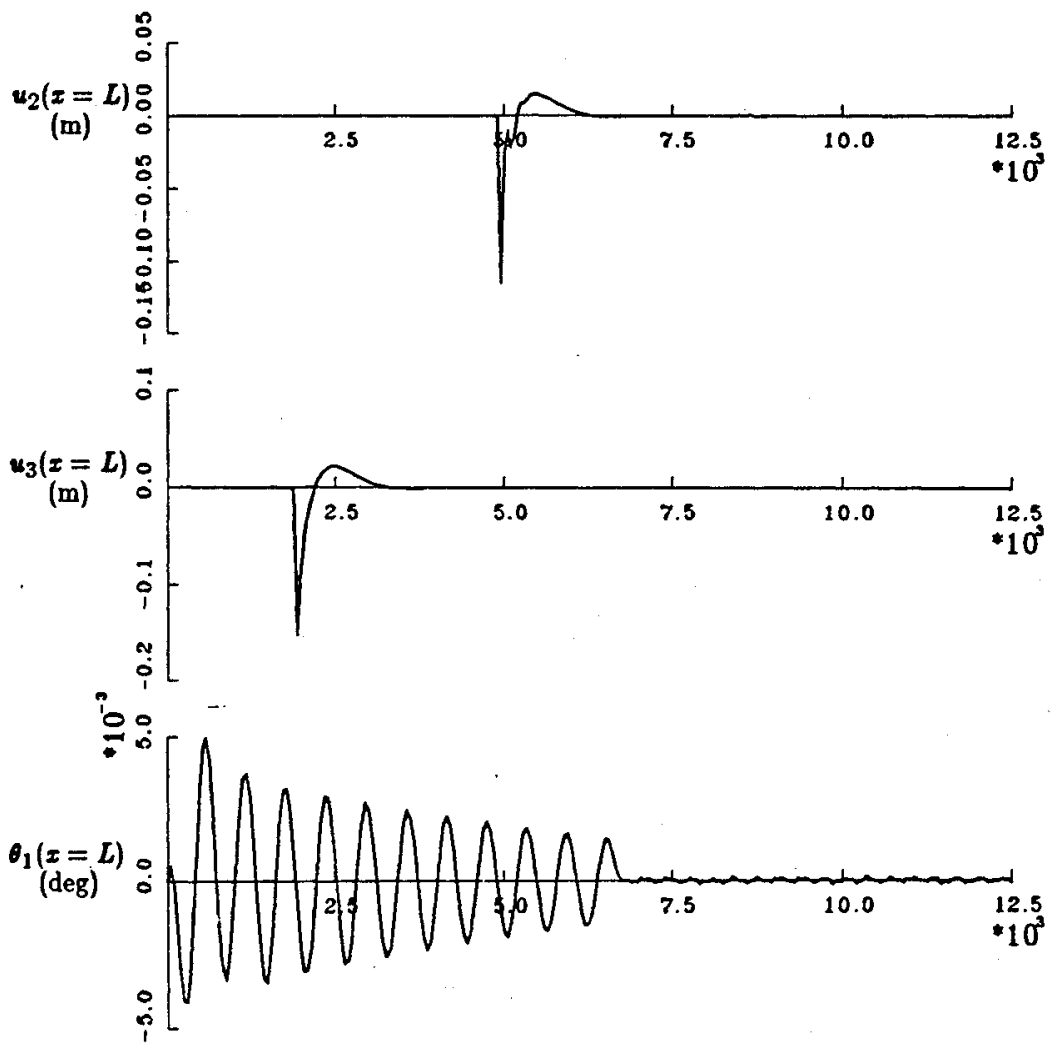

Time (sec)

$$
\begin{aligned}
& \mathscr{J}_{2} \dot{\omega}_{2}-\left(\mathscr{J}_{3}-\mathscr{J}_{1}\right) \omega_{3} \omega_{1}=T_{2}+G_{2} \\
& \mathscr{J}_{3} \dot{\omega}_{3}-\left(\mathscr{J}_{1}-\mathscr{J}_{2}\right) \omega_{1} \omega_{2}=T_{3}+G_{3}
\end{aligned}
$$

where $T_{i}$ and $G_{i}(i=1,2,3)$ represent the $a_{i}$ measure numbers of the control torques and gravity torques, respectively. In order to bring the satellite from its initial orientation to the desired final orientation in a specified time, the functions $T_{1}$, $T_{2}$, and $T_{3}$ must be carefully chosen. Assume that one can determine a set of body-three: 1-2-3 rotation angles ${ }^{9} \delta_{1}^{*}, \delta_{2}^{*}$, and $\delta_{3}^{*}$ such that if the satellite in its initial orientation relative to an orbiting reference frame were subjected successively to dextral rotations of amount $\delta_{1}^{*}$ about $\boldsymbol{a}_{1}, \delta_{2}^{*}$ about $\boldsymbol{a}_{2}$, and $\delta_{3}^{*}$ about $a_{3}$, it would end up in the desired final orientation relative to the same orbiting reference frame. The functions $T_{1}, T_{2}, T_{3}$ will be chosen in the form

$$
T_{i}=k_{i}\left(\delta_{i}^{*}-\delta_{i}\right)-\sigma_{i} \delta_{i}-G_{i} \quad(i=1,2,3)
$$

where $\delta_{1}, \delta_{2}$, and $\delta_{3}$ are body-three: 1-2-3 successive rotation angles describing the current orientation relative to the initial one, and $k_{i}$ and $\sigma_{i}$ are non-negative "proportional" and "derivative" control gains. If Eqs. (82-84) are linearized in the variables $\omega_{1}, \omega_{2}$, and $\omega_{3}$, and if $\dot{\omega}_{i}$ is approximated by $\ddot{\delta}_{i}$, then the governing attitude equations can be rewritten as (no summation convention employed)

$$
\mathscr{J}_{i} \ddot{\delta}_{i}+\sigma_{i} \dot{\delta}_{i}+k_{i}\left(\delta_{i}-\delta_{i}^{*}\right)=0 \quad(i=1,2,3)
$$

This is the standard form of the equation for a damped oscillator. Appropriate values for $\sigma_{i}$ and $k_{i}$ can thus be chosen to provide the desired combination of peak time, settling time, and overshoot. To assess the performance of such a control law for both a rigid and deformable satellite, consider the repositioning maneuver sequence illustrated in Fig. 9. The satellite is originally in an Earth-pointing geosynchronous orbit, and it is desired to reorient the satellite from this orientation to the final one shown in the sketch. The appropriate values of the body-three: 1-2-3 angles $\delta_{1}^{*}, \delta_{2}^{*}, \delta_{3}^{*}$, introduced earlier, are $90,-30$, and $20 \mathrm{deg}$, respectively. Note that these angles describe the desired orientation of the satellite relative to the inertial orientation that the satellite would have at the same orbit location if its attitude relative to the rotating orbital reference frame had remained undisturbed. The dotted-line curves in Fig. 10 show the angles $\delta_{1}, \delta_{2}, \delta_{3}$ as a function of time, these results having been obtained from a simulation in which $B$ was treated as rigid. Performing a subsequent simulation in which the deformation of $B$ is 
described by three transverse bending modes in each of the two principal transverse directions, as well as one torsional mode, the resulting values for $\delta_{1}, \delta_{2}, \delta_{3}$ appear as the solid lines in Fig. 10. Figure 11 displays the two transverse displacements and the twist of the beam tip during this maneuver.

The results indicate that whereas the control system succeeds in bringing about the desired reorientation, even when the satellite is flexible, the time it takes to complete the whole reorientation sequence is much greater and the corresponding cost involved in operating the control system is higher for the flexible satellite than for the rigid satellite, chiefly because the very strict tolerances on pointing could not be reached until after a considerable settling time of the beam vibrations.

It is important to state here that the controllers employed in the previous simulations were chosen in a simple way to clarify the key issues involved in the interaction between flexible appendages and rigid base motion. In practice, a more sophisticated approach to control law design and development would normally be employed in order to optimize the performance of the active control system. Such optimal control schemes can easily be incorporated in an algorithm based on the equations given here.

\section{Discussion and Conclusions}

This paper, in conjunction with Ref. 1 , provides a comprehensive theory and equations of motion for a free-flying rigid body with flexible beamlike appendages. The present theory has been compared with conventional modal modeling methods applied to beams and has been found to yield results that agree much more favorably with experimentally observed behavior. However, the increased accuracy of the results has been gained at the expense of generality. The conventional modal modeling method purports to treat any flexible component, whether it is composed of beams, plates, shells, or three-dimensional solids, whereas the present theory is applicable only to components and appendages that behave in a beamlike manner. Unfortunately, straightforward application of the conventional theory to any structural elements undergoing continuous large rotations yields inaccurate simulation results unless discretization or substructuring techniques are artificially performed. Naturally, the extent of the inaccuracy depends on the relative magnitude of the rate of change or orientation of the element and the flexural rigidities of the element. One valid conclusion that can be made is that in order to simulate accurately general aerospace structures undergoing large overall motions and small deformations (i.e., small strains), it is necessary to treat each distinct type of structural element constituting the system in a special way. Large rotation theories for beams, plates, and shells must be built into whatever system equation formulation procedure is employed, regardless of whether assumed mode techniques are used.

A natural question that often arises is: If it is necessary to incorporate these special structural theories in the dynamical formulation anyway, what advantage is there in using assumed mode methods as opposed to nonlinear finite-element methods? The following discussion is intended to address this question and shed light on practical implications of employing each method. First of all, a brief explanation of the differences between assumed mode methods and finite-element methods will be given.

The assumed mode method of treating flexible multibody systems undergoing large overall motions has been the most popular to date owing to a number of attractive features. First, one can easily take advantage of previously constructed structural finite-element models to provide eigenfunctions for the nonlinear simulation, thereby assuring some fidelity of models throughout the design and analysis process. Second, reducing the size of the system of equations for control design purposes is very easy to do with the assumed mode method by simply truncating the number of assumed modes employed.
Third, in many cases, good accuracy in simulation results can be obtained with a minimum of generalized temporal coordinates (and corresponding equations) because the assumed modal functions usually characterize the flexible-body deformation very well.

On the other hand, some of the advantages of the finiteelement method are that: 1) the piecewise continuous polynomial shape functions are easier to work with in the computational algorithm and cheaper to evaluate than their modal counterparts; 2) it is easier to allow for changes in boundary conditions of a flexible body (such as during deployment) with finite-element methods owing to the local nature of the shape functions and the standard global assembly procedures; and 3) an identical geometric model can be used in the nonlinear simulation as was used in earlier phases of design and structural analysis.

To give some idea of relative computational speed, CPU times on an Apollo DN3000 were monitored during the simulation reported in Fig. 5. Three analyses were performed: the first used the conventional assumed mode modeling method now employed in commercial software; the second was based on an algorithm employing the theory included in this paper; and the third used a fully nonlinear finite-element technique. As a benchmark, the present theory with 20 modes was used to establish the "correct solution."

The algorithm based on the assumed mode modeling theory included in this paper was able to reproduce the correct solution with $2 \%$ error in $381 \mathrm{CPU}$ seconds by employing only three transverse bending modes in the simulation. An algorithm based on the conventional assumed mode modeling approach, although not able to produce as accurate results (see Fig. 5), took only 299 CPU seconds with three modes to complete the simulation. The finite-element implementation, however, required nearly 10 elements to obtain comparable accuracy with that of the new modal method and used more than $6200 \mathrm{CPU}$ seconds. With three and five elements, respectively, the simulation times were 714 and 2557 CPU seconds, and the error was in the $5-10 \%$ range.

One reason for the unusually long simulation times with the finite-element implementation is that six degrees of freedom are allowed at each nodal point connecting the discrete element domains, whereas the simulations based on the assumed mode methods included flexibility in only one transverse direction. The unnecessary degrees of freedom could have been eliminated from the finite-element analysis; however, the absolute-coordinate, sparse-matrix analysis scheme employed would have required that additional constraint equations be processed at an expense greater than that of retaining all of the extra degrees of freedom in the problem.

Although the numerical results are by no means general or conclusive, they do suggest that it is worthwhile to pursue a general development of flexible multibody simulation algorithms that utilize the assumed mode procedures but also include large-rotation structural element theories.

\section{References}

${ }^{1}$ Kane, T. R., Ryan, R. R., and Banerjee, A. K., "Dynamics of a Cantilever Beam Attached to a Moving Base," Journal of Guidance, Control, and Dynamics, Vol. 10, March-April 1987, pp. 139-151.

${ }^{2}$ Bodley, C. S., Devers, A. D., Park, A. C., and Frisch, H. P., "A Digital Computer Program for the Dynamic Interaction Simulation of Controls and Structure (DISCOS)," NASA TP 1219, May 1978.

${ }^{3}$ Frisch, H. P., "A Vector-Dyadic Development of the Equations of Motion for N-Coupled Flexible Bodies and Point Masses." NASA TN D-8047, Aug. 1975.

${ }^{4}$ Ho, J. Y. L. and Herber, D. R., "Development of Dynamics and Control Simulation of Large Flexible Space Systems," Journal of Guidance, Control and Dynamics, Vol. 8, May-June 1985, pp. 374383.

${ }^{5}$ Singh, R. P., VanderVoort, R. J., and Likins, P. W., "Dynamics of Flexible Bodies in Tree Topology-A Computer Oriented Ap- 
proach," Proceedings of the AIAA/ASME/ASCE 25th Structures, Structural Dynamics and Materials Conference, AIAA, New York, May 1984.

${ }^{6}$ Yoo, W. S. and Haug, E. J., "Dynamics of Flexible Mechanical Systems Using Vibration and Static Correction Modes," American Society of Mechanical Engineers, Paper 85-DET-71, 1985.

${ }^{7}$ Oden, J. T. and Ripperger, E. A., Mechanics of Elastic Structures, 2nd ed., McGraw-Hill, New York, 1981.

${ }^{8}$ Roark, R. J. and Young, W. C., Formulas for Stress and Strain, 5th ed., McGraw-Hill, New York, 1975, pp. 5-12.

${ }^{9}$ Kane, T. R., Likins, P. W., and Levinson, D. A., Spacecraft Dynamics, McGraw-Hill, New York, 1983, pp. 87, 13, 61, 247, 425.

${ }^{10} \mathrm{Yu}, \mathrm{Y}$. Y., "Thermally Induced Vibration and Flutter of a Flexible Boom," Journal of Spacecraft and Rockets, Vol. 6, Aug. 1969, pp. 902-910.

"Vigneron, F. R. and Garrett, T. W., "Solar Induced Lateral Vibrations of Alouette Sounder Antennas During Extension," Defence Research Telecommunications Establishment, Ottawa, Rept. 1169, Dec. 1966
${ }^{12}$ Frisch, H. P., "Thermally Induced Vibrations of Long ThinWalled Cylinders of Open Section," Journal of Spacecraft and Rockets, Vol. 7, Aug. 1970, pp. 897-905.

${ }^{13}$ Lips, K. W., Graham, W. B., Vigneron, F. R., and Hunter, D. G., "Dynamics and Control Characteristics for the WISP 300m Dipole Antenna/Shuttle Configuration," American Astronautical Society, Paper 365, 1985.

${ }^{14}$ Simo, J. C., "A Finite Strain Beam Formulation. The ThreeDimensional Dynamic Problem. Part I," Computer Methods in Applied Mechanics and Engineering, Vol. 49, No. 1, 1985, pp. 55-70.

${ }^{15}$ Modi, V. J., "Attitude Dynamics of Satellites with Flexible Appendages-A Brief Review," Journal of Spacecraft, Vol. 11, Nov. 1974, pp. 743-751.

${ }^{16}$ Hughes, P. C. and Fung, J. C., "Liapunov Stability of Spinning Satellites with Large Flexible Appendages," Journal of Celestial Mechanics, Vol. 4, 1971, pp. 295-308.

${ }^{17}$ Kaza, K. R. V. and Kvaternik, R. G., "Nonlinear Flap-Lag-Axial Equations of a Rotating Beam," AIAA Journal, Vol. 15, June 1977, pp. $871-874$.

\author{
J. R. Bowen, J. C. Leyer, and R. I. Soloukhin, editors
}

Companion volumes, Dynamics of Explosions and Dynamics of Reactive Systems, I and II, cover new findings in the gasdynamics of flows associated with exothermic processing -the essential feature of detonation waves-and other, associated phenomena.

Dynamics of Explosions (volume 106) primarily concerns the interrelationship between the rate processes of energy deposition in a compressible medium and the concurrent nonsteady flow as it typically occurs in explosion phenomena. Dynamics of Reactive Systems (Volume 105, parts I and II) spans a broader area, encompassing the processes coupling the dynamics of fluid flow and molecular transformations in reactive media, occurring in any combustion system. The two volumes, in addition to embracing the usual topics of explosions, detonations, shock phenomena, and reactive flow, treat gasdynamic aspects of nonsteady flow in combustion, and the effects of turbulence and diagnostic techniques used to study combustion phenomena.

\author{
Dynamics of Explosions \\ 1986664 pp. illus., Hardback \\ ISBN 0-930403-15-0 \\ AlAA Members $\$ 49.95$ \\ Nonmembers $\$ 84.95$ \\ Order Number V-106
}

Dynamics of Reactive Systems I and II 1986900 pp. (2 vols.), illus. Hardback ISBN 0-930403-14-2 AIAA Members $\$ 79.95$ Nonmembers $\$ 125.00$ Order Number V-105

TO ORDER: Write, Phone, or FAX: AIAA c/O TASCO,

9 Jay Gould Ct., P.O. Box 753, Waldorf, MD 20604

Phone (301) 645-5643, Dept. 415 — FAX (301) 843-0159

Sales Tax: CA residents, 7\%; DC, $6 \%$. Add $\$ 4.75$ for shipping and handling of 1 to 4 books (Call for rates on higher quantities). Orders under $\$ 50.00$ must be prepaid. Foreign orders must be prepaid. Please allow 4 weeks for delivery. Prices are subject to change without notice. Returns will be accepted within 15 days. 